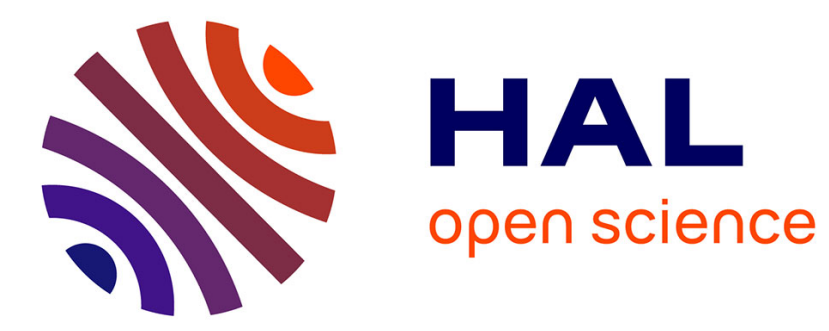

\title{
Modeling of pyrolysis in a high capacity thermo balance
} Frederic Marias, Stephanie Delage Santacreu

\section{To cite this version:}

Frederic Marias, Stephanie Delage Santacreu. Modeling of pyrolysis in a high capacity thermo balance. Canadian Journal of Chemical Engineering, 2014, 93 (2), pp.261-275. 10.1002/cjce.22084. hal02159178

\section{HAL Id: hal-02159178 https://hal-univ-pau.archives-ouvertes.fr/hal-02159178}

Submitted on 28 Nov 2019

HAL is a multi-disciplinary open access archive for the deposit and dissemination of scientific research documents, whether they are published or not. The documents may come from teaching and research institutions in France or abroad, or from public or private research centers.
L'archive ouverte pluridisciplinaire HAL, est destinée au dépôt et à la diffusion de documents scientifiques de niveau recherche, publiés ou non, émanant des établissements d'enseignement et de recherche français ou étrangers, des laboratoires publics ou privés. 


\section{INTRODUCTION}

$I^{\infty}$

This paper deals with the two dimensional modelling of transient pyrolysis of biomass in a fixed bed. The model which is developed mainly relies on the theory of volume averaging. This model includes heat and mass transfer and pyrolysis reaction. It allows the prediction of temperature, moisture content organic material content, and char content, as well as the prediction of internal evolution of the composition of the gas held and released from the sample under investigation.

This model is used in order to describe pine wood pyrolysis taking place in a macro thermobalance which is also presented in the paper. Comparison of the numerical results with the experimental ones, in terms of mass loss of the sample, shows good agreement and validates the model. A deeper analysis of the results allows a better understanding of the processes involved in the thermal degradation.

Keywords: modelling, pyrolysis, porous media, high capacity thermobalance the frame of the renewal of our energy mix, there is evidence on the fact that biomass is liable to play a major role in (composed of several compounds) should either burnt to provide heat, pyrolysed to provide liquid, gas and/or solid fuel, or gasified to produce gas. ${ }^{[1,2]}$ However, both of them involve a pyrolysis step (in combustion process, there is a preliminary pyrolysis step where the fresh biomass is converted to volatiles and char which are further oxidized by homogeneous and heterogeneous reactions respectively) and this step is at the heart of biomass conversion.

This solid thermal degradation in the absence of oxidizing agent, results in the production of a huge number of chemical compounds which are often lumped into three groups: a permanent gas, a gas condensed at ambient temperature (tars) and a solid residue (char). ${ }^{[3,4]}$ The yield of each of these groups is highly dependent on temperature and heating rate. ${ }^{[3,5-7]}$ Processes are often separated into flash pyrolysis (high or very high heating rate and temperature above $550^{\circ} \mathrm{C}$ ) and slow pyrolysis (heating rate of few tens of ${ }^{\circ} \mathrm{C} / \mathrm{min}$ at temperature lower than $500^{\circ} \mathrm{C}$ ).

For both processes, there is a huge work that has been done in order to determine the products of the operation (in terms of permanent gas and tar composition) as well as the associated kinetics. ${ }^{[8-18]}$

The present work focusses on this slow pyrolysis. Indeed, as it will be shown, heating rate is about $10^{\circ} \mathrm{C} / \mathrm{min}$ while the temperature at which the sample is raised is lower than $300^{\circ} \mathrm{C}$. For this kind of conditions, it is well known that pyrolysis of biomass mainly leads to char and to a lower extent, to tars and noncondensable gases. ${ }^{[1-3,19-20]}$ In this work, a mathematical model is developed in order to get insights on the slow pyrolysis of biomass in a fixed bed. This model is based on the volume averaging theory ${ }^{[21]}$ which allows translating the heterogeneous medium under investigation into a continuous one. In the present version of this work, this model is used assuming a three parallel reactions mechanism for pyrolysis. The results of the model are compared with experimental data obtained in a high capacity thermobalance which is presented in the first part of the paper. Then, the mathematical model is derived and followed by a presentation of the obtained results.

\section{EXPERIMENTAL SETUP}

The device under study is depicted in Figure 1. Conceptually, it is equivalent to a commercial thermobalance, but it can accept a load of up to $1 \mathrm{~kg}$ of biomass, depending upon its density. Basically, the device is firstly composed of a furnace (capacity 751) that can raise the temperature of its content up to $850^{\circ} \mathrm{C}$. This commercial furnace has been made airtight in order to avoid leakages of volatile matter produced during pyrolysis, and in order to quantify this production. The pressure inside the device is limited to $150 \mathrm{kPa}$ (abs) and it is fixed by the pressure drop inside the tubes and the filter that connect the exhaust of the furnace to the atmosphere. A basket (Bk 101) is placed within the furnace which receives the load to be pyrolysed. This basket is cylindrical in shape, with an internal diameter of $15 \mathrm{~cm}$ and a height of $10 \mathrm{~cm}$. The lateral walls of this basket are made of stainless steel while its bottom is a made of a grid with a square arrangement (each square has a size of $2.5 \mathrm{~mm}$ ). This grid allows the nitrogen entering the furnace to flow inside the basket, within the sample to be pyrolysed itself. The basket is supported by a balance (Wg 101) placed outside of the furnace in a tight box, cooled by a nitrogen flow which in turn enters the furnace through the legs of the basket that establish the mechanical connection between it and the balance. This balance allows the recording of the mass of the sample held within the basket during the experiment. The furnace itself is also fed with a nitrogen flow in order to drag the product of pyrolysis outside of the furnace. The overall flow rate of nitrogen entering the device is

\footnotetext{
*Author to whom correspondence may be addressed. E-mail address: frederic.marias@univ-pau.fr Can. J. Chem. Eng. 9999:1-15, 2014 (C) 2014 Canadian Society for Chemical Engineering DOI 10.1002/cjce.22084

Published online in Wiley Online Library

(wileyonlinelibrary.com).
} 


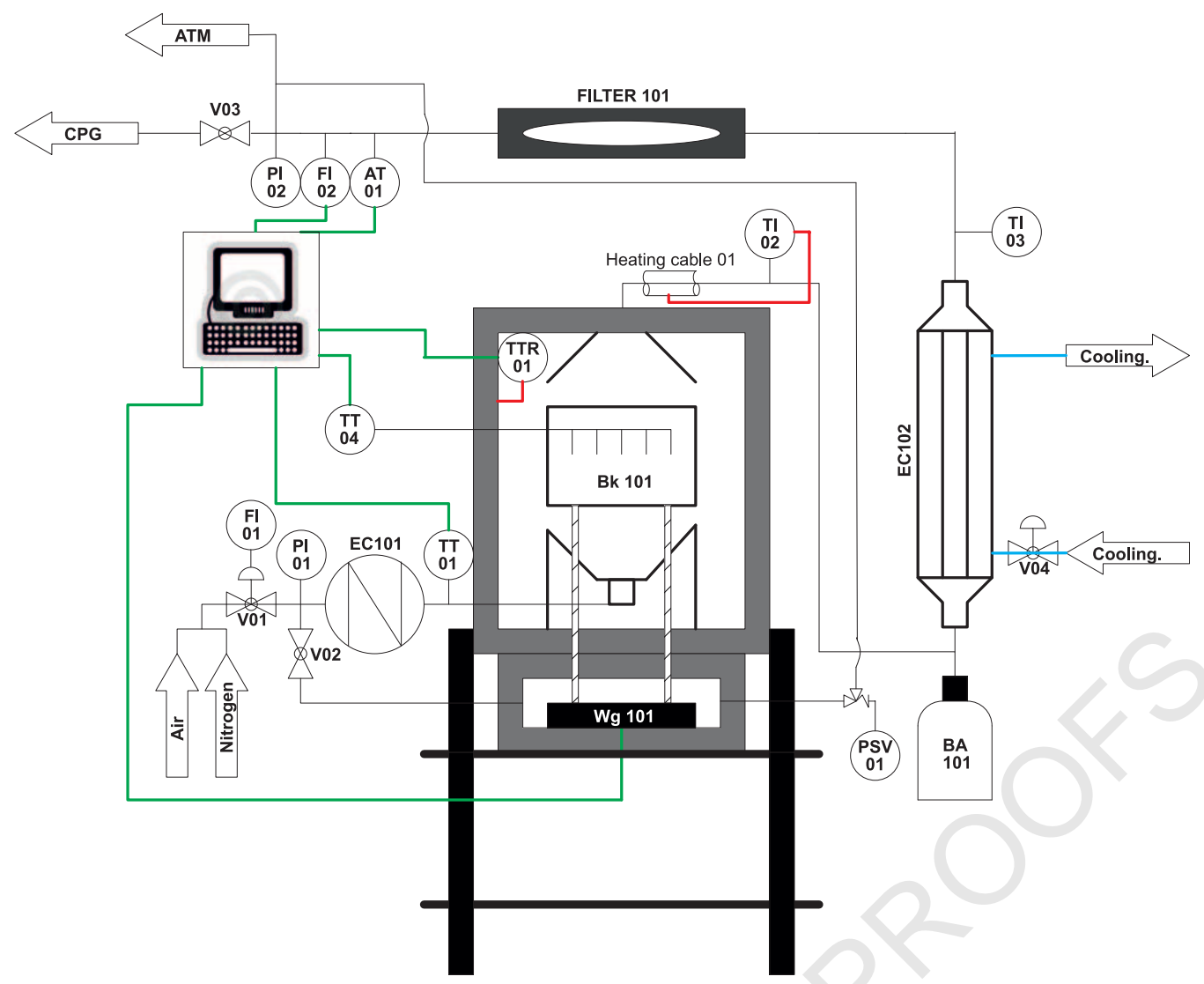

Figure 1. Scheme of the device under investigation.

quantified thanks to flowmeter FI 01 and controlled thanks to valve V01. Heater EC101 allows a potential heating of this nitrogen up to $750^{\circ} \mathrm{C}$. The gas leaving the furnace (composed of the incoming nitrogen and the products of pyrolysis) leaves the furnace in a pipe (heated to $250^{\circ} \mathrm{C}$ thanks to an electrical heating cable) and then it enters a condenser (EC102) where tars are condensed and collected in receptacle BA 101. After the condensing stage, the resulting gas is routed towards a filter (Filter 101 filled with cotton) where aerosols are separated from the gas and collected. Finally, the gas is analyzed (gaseous chromatography) and its flow rate is quantified (FI02). Thermocouple TTR01, located on the roof of the furnace is used to measure and control the temperature inside the furnace, while the thermocouples TT01, TI02, TI03 and TT04 allow a monitoring of the values of temperature at different positions in the overall device. Figure 2 shows two photos of the overall equipment. More details about this device can be found in. ${ }^{[27]}$

\section{MATHEMATICAL MODEL}

Because of the important size of the sample $(15 \mathrm{~cm}$ diameter, and $10 \mathrm{~cm}$ high), this one should not be considered as homogeneous while the pyrolysis process takes place. Indeed, because heat and mass transfer within the sample strongly influence the rate at which the initial biomass is firstly dried and then pyrolysed these last "reactions" phenomena should not be considered as processing at the same rate at each location in the sample. This is the reason

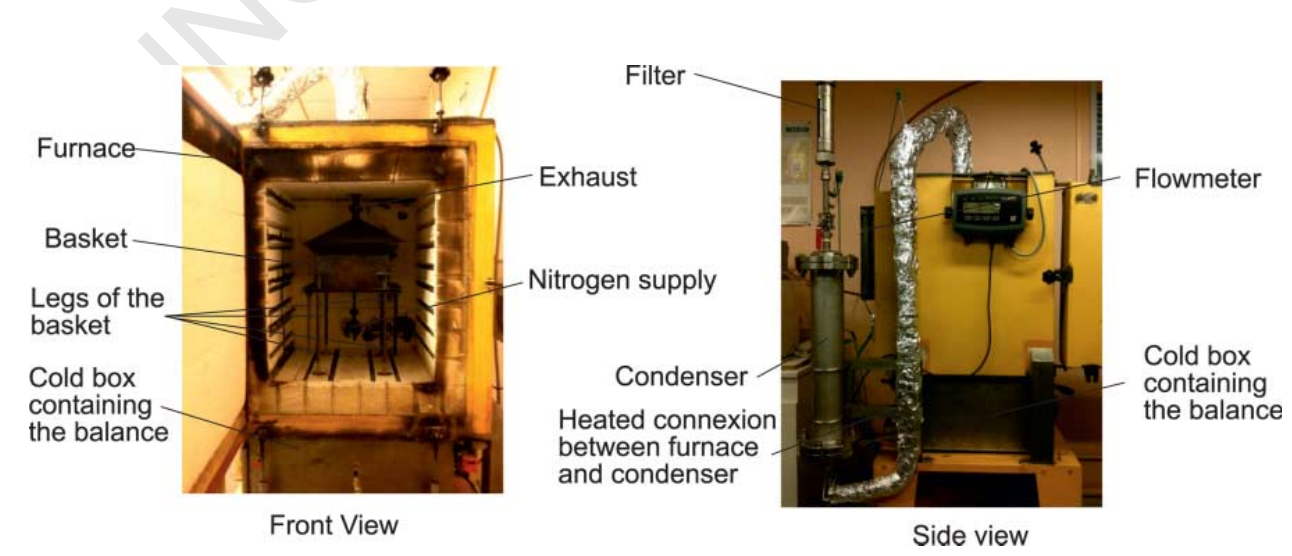

Figure 2. Photos of the high capacity thermobalance (Top: front view, bottom: side view). 
why, and to our opinion, a description taking into account this heterogeneity (in terms of temperature and composition) must be accounted for in the modelling effort. It must also be pointed out, that such heterogeneity could also occur within industrial reactors (downdraft or updraft pyrolysers), and hence, models relying on $2 \mathrm{D}$ description of the moving bed of solids should be used. Moreover, because biomass is strongly heterogeneous in nature (porous material), a proper description of the processes that lead to its conversion should take into account this heterogeneity and not consider the material as a unique phase. This is the reason why the mathematical model which has been developed here is based on the concept of volume averaging. Hence, in a first step, a simplified description of this concept will be proposed. In a second step, the description of the system under consideration (from the physical point of view) will be stated, and finally, the equations constituting the mathematical model of this system will be derived.

\section{Basics on Volume Averaging Theory}

This theory was first proposed by Whitaker ${ }^{[21]}$ in a work devoted to the drying of porous media. Then, It has been widely used in different fields associated with heat and mass transfers in reactive or non-reactive porous media (Biofilms, ${ }^{[22]}$ Catalytic reaction, ${ }^{[23]}$ Combustion ${ }^{[24]}$ ) and also in the field of pyrolysis. ${ }^{[25,26]}$ This theory consists in the homogenization of properties defined for each phase of the medium under consideration at its "phase scale" over a volume where the medium can be described by equivalent properties, continuous over this volume. As an example, Figure 3 sketches a porous medium composed of three phases (solid, liquid and gas). Over each of this continuous phase, it is possible to derive conservation equations (mass, momentum, energy) using the fundamentals of continuum mechanics. However, integrating these equations over the complete system (which can reach the size of an industrial reactor) is impossible because it would require the exact geometry of the porous medium essential to quantify exchanges between phases. Hence, the concept is to make a preintegration, over an averaging volume, where the properties of the medium are lumped into averaged properties. More precisely, if $\psi_{\alpha}$ is a property defined in phase $\alpha$ (which can be either Temperature, density, pressure, density....), its average value over the averaging volume $V$ is defined as:

$\left\langle\psi_{\alpha}\right\rangle=\frac{1}{V} \int_{V_{\alpha}} \psi_{\alpha} d V^{\prime}$

Where $V_{\alpha}$ stands for the volume occupied by the phase $\alpha$ within $V$.

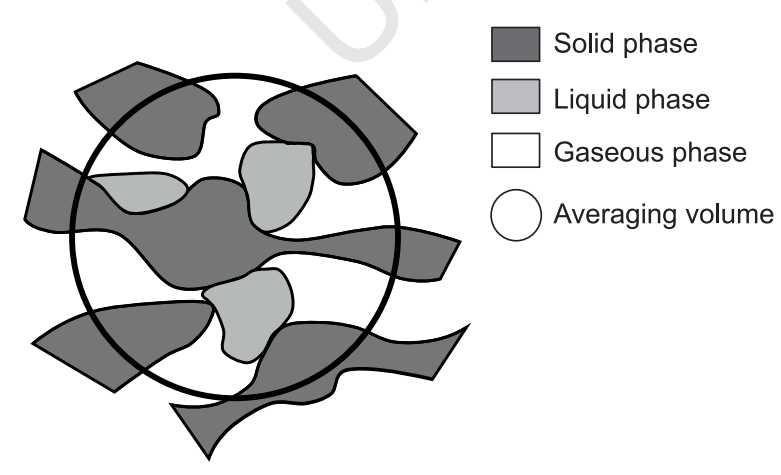

Figure 3. Scheme of a porous medium composed of three phases.
It is also required to define the intrinsic phase average, as follows:

$\left\langle\psi_{\alpha}\right\rangle^{\alpha}=\frac{1}{V_{\alpha}} \int_{V_{\alpha}} \psi_{\alpha} d V^{\prime}$

Both equations are linked through the volume fraction of phase $\alpha, \varepsilon_{\alpha}$ defined by:

$\varepsilon_{\alpha}=V_{\alpha} / V$

$\left\langle\psi_{\alpha}\right\rangle=\varepsilon_{\alpha}\left\langle\psi_{\alpha}\right\rangle^{\alpha}$

A two-step method is applied. The first one consists in derivating the equations at the phase scale (where continuum mechanics applies). In the second step, these equations are averaged over the averaging volume, in order to obtain equations that link together the average properties.

\section{Description of the System Under Consideration}

This study deals with biomass pyrolysis. The considered system must allow the translation into mathematical formalism of the main processes involved during pyrolysis.

More generally, biomass can be considered as a mixture of organic material, inorganic material (which becomes ash when the biomass is burnt), and moisture. Of course, the porous bed composed of the particles of the biomass held within the basket of the macro thermobalance can be considered as a porous medium, where the open porosity is filled with gas. This gas is air saturated with water vapor, when the biomass is at rest. It is thus required that the description of the system take into account these first four phases: Organic matter, inorganic matter, liquid (moisture) and gas (which is a multicomponent mixture).

As the sample is heated, it first undergoes a step of drying where liquid water is converted into vapor. More precisely, above the fiber saturation point, which is an intrinsic characteristic of the considered material, water is expected to be free of interactions with the solid matrix. Hence its evaporation solely requires its latent heat of vaporization as energy input. Below this saturation point, water is linked to the solid material and extra energy (above the latent heat of vaporization of pure water) is required for its desorption. As a result, the liquid phase has to be split into "free" and "bound" water. Although liquid phases may not only be composed of pure water, this assumption is used in the frame of this study.

After the drying step, the temperature of the sample is raising again until the pyrolysis process occur.

A lot of work has been done in order to characterize the reaction pathway and the possible products of the reaction. ${ }^{[2-32]}$ It is known that this process, associated with the decomposition of organic material under the effect of heat, leads to the formation of products, that can be grouped into several categories: two solid fractions (commonly called "intermediate" and "char"), a gas fraction that could be condensed at ambient conditions (referred as tars) and a gas fraction that could not be condensed at ambient conditions (permanent). This first decomposition (primary pyrolysis) might be followed by heterogeneous reaction between the products of primary pyrolysis and char (with possible catalytic effect of the inorganic material), homogenous reaction in the gas phase and possible condensation and evaporation of tars. This second set of processes is commonly referred as secondary 


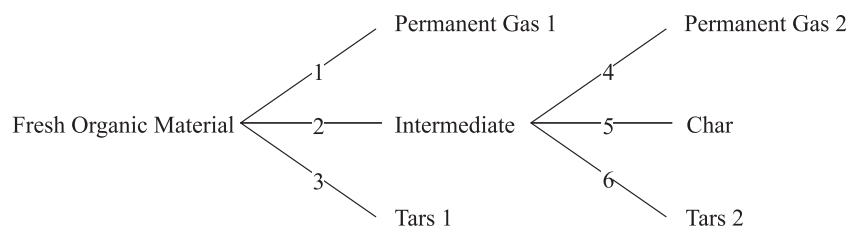

Figure 4. Scheme of the chemical pathway used in the model.

pyrolysis. However, in the frame of this study, which is restricted to torrefaction, the temperature of the sample should not exceed $300{ }^{\circ} \mathrm{C}$. This is the reason why, the secondary phenomena have not been taken into account.

In order to cover the overall set of chemical pathway that has been published in literature and in accordance with the work of $C$. Casajus, ${ }^{[32]}$ the chemical pathway depicted in Figure 4 has been used in our model.

As a sum up of this paragraph, it can be stated that the description of our sample includes

- Four solid phases (fresh organic material, intermediate and char and inorganic material)

- Two liquid phases (free and bound pure water)

- A gaseous phase (which is a multi-component mixture).

Dealing with this last phase, it is required to define the nature of the species that can be present. In some experimental work on the above mentioned device, $C$. Casajus ${ }^{[32]}$ showed that this mixture was mainly composed of Methane, Hydrogen, Carbon monoxide and carbon dioxide as permanent gases, and, water, Acetic acid, Lactic acid, formic acid, hydroxyaceton, acetaldehyde, formaldehyde, methanol and furfural. Given the fact that the initial sample contains air, and that nitrogen is provided to the furnace in order to keep inert conditions and to evacuate the products of pyrolysis during its occurrence, both oxygen and nitrogen have been added to the list of species that can be present in the gas phase. This means that the gaseous phase is composed of NS $=15$ species.

\section{Mathematical Model}

In this section, the mathematical model, that translates the physical and chemical phenomena occurring within the device into mathematical formalism, is established. Given the physical description of the system that was provided in the preceding section, the first paragraph of present section will focus on the determination of the state variables of the system. These state variables are computed by the model and allow a complete description of the sample at any time and any position. Then, given this set of variables, the next section will be devoted to the presentation of the average equations of conservation of mass, species, energy and momentum. Finally, in the third section the constitutive equations will be provided.

\section{State variables}

This section of the work is devoted to the description of the variables that need to be computed in order to characterize the state of the material at each time. These variables can be summarized as:

- $\langle T\rangle$ average temperature of the sample held in the basket (one temperature model, gas, liquid and bound water, organic matter and inorganic matter have the same temperature)

- $\left\langle P_{g}\right\rangle^{g}$ intrinsic gas average pressure inside the porous media

- $\left\langle\rho_{g}\right\rangle^{g}$ intrinsic gas average mass concentration in the porous media
- $\left\langle\rho_{i}\right\rangle^{g}$ intrinsic gas average mass concentration of species $i$ in the porous media (15 species)

- $\left\langle\rho_{l}\right\rangle$ average free water mass concentration in the porous media

- $\left\langle\rho_{b}\right\rangle$ average bound water mass concentration in the porous media

- $\left\langle\rho_{O M}\right\rangle$ average organic matter mass concentration in the porous media

- $\left\langle\rho_{\text {Int }}\right\rangle$ average intermediate mass concentration in the porous media

- $\left\langle\rho_{I N O}\right\rangle$ average inorganic matter mass concentration in the porous media

- $\left\langle\rho_{C}\right\rangle$ average intermediate mass concentration in the porous media

- $\varepsilon_{g}$ volume fraction of the gas phase

\section{Balance equations}

This section of the paper is devoted to the description of the equations used in order to compute the above mentioned variables. It has to be noticed here that these equations result from a first derivation of the relevant equations at the phase scale and a subsequent mathematical averaging. This mathematical procedure has not been included in the paper in order to make it shorter. Instead, we have chosen to present the final equations that are solved by the numerical procedure. Readers interested in the averaging procedure should refer to ${ }^{[21]}$ and $^{[33]}$

Mass conservation of organic matter.

$$
\frac{\partial}{\partial t}\left\langle\rho_{O M}\right\rangle=\left\langle\dot{w}_{O M}\right\rangle
$$

This equation states that accumulation of average organic material mass concentration is balanced by its rate of pyrolysis reaction which is computed thanks to the scheme proposed in Figure 4

$$
\left\langle\dot{w}_{O M}\right\rangle=-k_{1}\left\langle\rho_{O M}\right\rangle^{n_{1}}-k_{2}\left\langle\rho_{O M}\right\rangle^{n_{2}}-k_{3}\left\langle\rho_{O M}\right\rangle^{n_{3}}
$$

Where $k_{1}, k_{2}$, and $k_{3}$ respectively stands for the average of the kinetic constant (depending upon the average temperature) involved in the three parallel reaction schemes involving the decomposition of organic material, and $n_{1}, n_{2}$ and $n_{3}$ the corresponding reaction orders. It has to be emphasized here that, because the material has been assumed to keep its original shape (no shrinkage) the equation does not contain any convection term that should be included if shape modification had been taken into account.

Mass conservation of intermediate. This equation is quite similar to the preceding one:

$$
\frac{\partial}{\partial t}\left\langle\rho_{\text {Int }}\right\rangle=\left\langle\dot{w}_{\text {Int }}\right\rangle
$$

However, because of the chemical reaction pathway used in the frame of this study, the reaction rate occurring in this equation is evaluated according to:

$$
\left\langle\dot{w}_{\text {Int }}\right\rangle=+k_{2}\left\langle\rho_{\text {OM }}\right\rangle^{n_{2}}-k_{4}\left\langle\rho_{\text {Int }}\right\rangle^{n_{4}}-k_{5}\left\langle\rho_{\text {Int }}\right\rangle^{n_{5}}-k_{6}\left\langle\rho_{\text {Int }}\right\rangle^{n_{6}}
$$

Mass conservation of inorganic matter.

$\frac{\partial}{\partial t}\left\langle\rho_{I N O}\right\rangle=0$ 
Given the fact that inorganic material is supposed to be inert and that there is no modification of shape in the medium under investigation, Equation (9) simply states that there is no accumulation (positive or negative) of this material in the medium.

Mass conservation of char.

$\frac{\partial}{\partial t}\left\langle\rho_{C}\right\rangle=\left\langle\dot{w}_{C}\right\rangle$

In a similar manner to organic material, char might be produced by the heterogeneous pyrolysis reaction. Hence, its accumulation within the average volume under study is balanced by its chemical reaction rate which is further computed according to the above mentioned described reaction scheme:

$\left\langle\dot{w}_{C}\right\rangle=k_{5}\left\langle\rho_{\text {Int }}\right\rangle^{n_{5}}$

Mass conservation of liquid water. Liquid water is assumed to be composed of pure water. Its average accumulation is balanced by convection in both directions and by production within the average volume which gives the following equation

$\frac{\partial}{\partial t}\left\langle\rho_{l}\right\rangle+\frac{1}{r} \frac{\partial}{\partial r}\left(r\left\langle\rho_{l}\right\rangle^{l}\left\langle v_{l}\right\rangle_{r}\right)+\frac{\partial}{\partial z}\left(\left\langle\rho_{l}\right\rangle^{l}\left\langle v_{l}\right\rangle_{z}\right)=\left\langle\dot{w}_{l}\right\rangle$

where $\left\langle v_{l}\right\rangle_{\mathrm{r}}$ and $\left\langle v_{l}\right\rangle_{\mathrm{z}}$ respectively stands for the average liquid velocity in the two directions under investigation (radius and height). These data are computed using the Darcy's law

$\left\langle v_{l}\right\rangle_{r}=-\frac{K_{l} K_{l}^{r}}{\mu_{l}} \frac{\partial}{\partial r}\left\langle P_{l}\right\rangle^{l}$

$\left\langle v_{l}\right\rangle_{z}=-\frac{K_{l} K_{l}^{r}}{\mu_{l}} \frac{\partial}{\partial z}\left\langle P_{l}\right\rangle^{l}$

where, $\left\langle P_{l}\right\rangle^{l}$ represents the intrinsic average liquid pressure in the volume under investigation and is computed as a function of the intrinsic average gaseous pressure $\left(\left\langle P_{g}\right\rangle^{g}\right)$ and the capillary pressure $\left(P_{C}\right)$ which depends upon the local total average moisture content and is computed according to a constitutive equation (see below)

$\left\langle P_{l}\right\rangle^{l}=\left\langle P_{g}\right\rangle^{g}-P_{C}$

In Equation (12), $\left\langle\dot{w}_{l}\right\rangle$ stands for the average rate of condensation or evaporation of the liquid water. It should be noticed here that no liquid water is expected to be directly produced by the pyrolysis reaction, but could be produced at the gaseous state and further condensed.

Mass conservation of bound water. In the case of bound water, the balance Equation (16) is very close to the one for liquid water (12)

$\frac{\partial}{\partial t}\left\langle\rho_{b}\right\rangle+\frac{1}{r} \frac{\partial}{\partial r}\left(r\left\langle\rho_{b} v_{b}\right\rangle_{r}\right)+\frac{\partial}{\partial z}\left(\left\langle\rho_{b} v_{b}\right\rangle_{z}\right)=\left\langle\dot{w}_{b}\right\rangle$

Given the fact that, the convective transport of this material cannot be correlated to the intrinsic average gaseous pressure, it is computed via a pseudo diffusion law, involving the local mass fraction of bound water over the total mass of dry solid. More precisely, the convective terms are evaluated as follows: $\left\langle\rho_{b} v_{b}\right\rangle_{r}=-\left\langle\rho_{S D}\right\rangle D_{b} \frac{\partial}{\partial r} \frac{\left\langle\rho_{b}\right\rangle}{\left\langle\rho_{S D}\right\rangle}$

$\left\langle\rho_{b} v_{b}\right\rangle_{z}=-\left\langle\rho_{S D}\right\rangle D_{b} \frac{\partial}{\partial z} \frac{\left\langle\rho_{b}\right\rangle}{\left\langle\rho_{S D}\right\rangle}$

where $\left\langle\rho_{S D}\right\rangle$ stands for the average mass concentration of the solid material (including organic material, inorganic material and char) over the volume under investigation. In Equation (16), $\left\langle\dot{w}_{b}\right\rangle$ represents the rate of desorption or adsorption of bound water.

However, there is no direct law to differentiate or to evaluate directly the rate of evaporation of bound or liquid water. Therefore Equations (12) and (16) are summed to obtain the so called balance equation for liquid + bound water whose solving is possible:

$$
\begin{gathered}
\frac{\partial}{\partial t}\left(\left\langle\rho_{b}\right\rangle+\left\langle\rho_{l}\right\rangle\right)+\frac{1}{r} \frac{\partial}{\partial r}\left(r\left(\left\langle\rho_{b} v_{b}\right\rangle_{r}+\left\langle\rho_{l} v_{l}\right\rangle_{r}\right)\right) \\
+\frac{\partial}{\partial z}\left(\left\langle\rho_{b} v_{b}\right\rangle_{z}+\left\langle\rho_{l} v_{l}\right\rangle_{z}\right)=\left\langle\dot{w}_{b}\right\rangle+\left\langle\dot{w}_{l}\right\rangle
\end{gathered}
$$

Mass conservation of component $i$ in the Gas mixture. The general equation for gaseous species conservation can be firstly written as it follows (20).

$\frac{\partial}{\partial t}\left(\varepsilon_{g}\left\langle\rho_{i}\right\rangle^{g}\right)+\frac{1}{r} \frac{\partial}{\partial r}\left(r\left\langle\rho_{i} v_{i}\right\rangle_{r}\right)+\frac{\partial}{\partial z}\left(\left\langle\rho_{i} v_{i}\right\rangle_{z}\right)=\left\langle\dot{w}_{i}\right\rangle$

where, $\varepsilon_{g}$ represents the fraction of the volume under investigation which is occupied by gas. The transport of gaseous species within this volume is the sum of a pure convective term and of a diffusive term:

$\left\langle\rho_{i} v_{i}\right\rangle_{r}=\left\langle\rho_{i}\right\rangle^{g}\left\langle v_{g}\right\rangle_{r}+\left\langle\rho_{i} U_{i}\right\rangle_{r}$

$\left\langle\rho_{i} v_{i}\right\rangle_{z}=\left\langle\rho_{i}\right\rangle^{g}\left\langle v_{g}\right\rangle_{z}+\left\langle\rho_{i} U_{i}\right\rangle_{z}$

Dealing with the pure convective term, it is correlated to the gradient of intrinsic average gaseous pressure through the Darcy's law:

$$
\begin{aligned}
& \left\langle v_{g}\right\rangle_{r}=-\frac{K_{g} K_{g}^{r}}{\mu_{g}} \frac{\partial}{\partial r}\left\langle P_{g}\right\rangle^{g} \\
& \left\langle v_{g}\right\rangle_{z}=-\frac{K_{g} K_{g}^{r}}{\mu_{g}} \frac{\partial}{\partial z}\left\langle P_{g}\right\rangle^{g}
\end{aligned}
$$

In the previous equation, $K_{g}$ (respectively $K_{g}^{r}$ ) stands for the intrinsic (respectively relative) permeability of the material under investigation, while $\mu_{g}$ represents the viscosity of the gaseous medium.

The diffusion terms are estimated using the Fick's Law:

$$
\begin{aligned}
& \left\langle\rho_{i} U_{i}\right\rangle_{r}=-\left\langle\rho_{g}\right\rangle^{g} D_{i} \frac{\partial}{\partial r} \frac{\left\langle\rho_{i}\right\rangle^{g}}{\left\langle\rho_{g}\right\rangle^{g}} \\
& \left\langle\rho_{i} U_{i}\right\rangle_{z}=-\left\langle\rho_{g}\right\rangle^{g} D_{i} \frac{\partial}{\partial z} \frac{\left\langle\rho_{i}\right\rangle^{g}}{\left\langle\rho_{g}\right\rangle^{g}}
\end{aligned}
$$

where $D_{i}$ stands for the diffusion coefficient of species $i$ within the gaseous mixture. 
Finally, Equation (20) requires the rate of production of species $i$ within the considered volume. Because we focus in this work on primary pyrolysis, this term is only associated to heterogeneous pyrolysis reaction and, in the case of water, to this reaction and change of state from liquid or bound water to vapour water.

Dealing with the pyrolysis reaction, gaseous species can be produced either by the reaction leading to permanent gases or by
This equation implies transport terms associated to the transport of liquid and bound water, and gaseous species. No more details are given about these terms which have been discussed in the previous equations.

This equation also includes transport of heat by conduction in both directions with an effective conductivity $k_{\text {eff }}$ evaluated through correlations that will be given later in the paper.

$$
\begin{aligned}
& \frac{\partial\left(\varepsilon_{g}\left\langle\rho_{g}\right\rangle^{g} C_{p, g}+\left\langle\rho_{l}\right\rangle C_{p, l}+\left\langle\rho_{b}\right\rangle C_{p, l}+\left\langle\rho_{\text {OM }}\right\rangle C_{p, O M}+\left\langle\rho_{\text {Int }}\right\rangle C_{p, I n t}+\left\langle\rho_{\text {INO }}\right\rangle C_{p, I N O}+\left\langle\rho_{C}\right\rangle C_{p, C}\right)\langle T\rangle}{\partial t} \\
& +\frac{1}{r} \frac{\partial}{\partial r}\left(r\left(\left\langle\rho_{l}\right\rangle^{l}\left\langle v_{l}\right\rangle_{r} C_{p, l}+\left\langle\rho_{b} v_{b}\right\rangle_{r} C_{p, l}+\left\langle\rho_{g} v_{g}\right\rangle_{r} C_{p, g}+\sum_{i=1}^{N_{S}} C_{p, g}\left\langle\rho_{i} U_{i}\right\rangle_{r}\right)\langle T\rangle\right) \\
& +\frac{\partial}{\partial z}\left(\left\langle\rho_{l}\right\rangle^{l}\left\langle v_{l}\right\rangle_{z} C_{p, l}+\left\langle\rho_{b} v_{b}\right\rangle_{z} C_{p, l}+\left\langle\rho_{g} v_{g}\right\rangle_{z} C_{p, g}+\sum_{i=1}^{N_{S}} C_{p, g}\left\langle\rho_{i} U_{i}\right\rangle_{z}\right)\langle T\rangle \\
& =\frac{1}{r} \frac{\partial}{\partial r}\left(r k_{e f f} \frac{\partial}{\partial r}\langle T\rangle\right)+\frac{\partial}{\partial z}\left(k_{e f f} \frac{\partial}{\partial z}\langle T\rangle\right)-\left\langle\dot{w}_{O M}\right\rangle \Delta h_{O M}-\left\langle\dot{w}_{\text {Int }}\right\rangle \Delta h_{\text {Int }}+\left\langle\dot{w}_{H_{2} O}\right\rangle^{v a p} \Delta h_{v a p}
\end{aligned}
$$

the reaction leading to condensable gases. Hence the associated production term is computed as:

$$
\begin{aligned}
\left\langle\dot{w}_{i}\right\rangle^{\text {pyr }}= & k_{1} Y_{i}^{\text {perm }, 1}\left\langle\rho_{\text {OM }}\right\rangle^{n_{1}}+k_{3} Y_{i}^{\text {tar }, 1}\left\langle\rho_{\text {OM }}\right\rangle^{n_{3}} \\
& +k_{4} Y_{i}^{\text {perm }, 2}\left\langle\rho_{\text {Int }}\right\rangle^{n_{4}}+k_{6} Y_{i}^{\text {tar }, 2}\left\langle\rho_{\text {Int }}\right\rangle^{n_{6}}
\end{aligned}
$$

In Equation (27), $Y_{i}^{\text {perm,1 }}$ and $Y_{i}^{\text {perm,2 }}$ represent respectively the mass fraction of species $i$ released, as a permanent gas, during the pyrolysis reactions 1 and 4 , while $Y_{i}^{\text {tar,1 }}$ and $Y_{i}^{\text {tar,2 }}$ represent respectively the mass fraction of species $i$ released, as a condensable gas, during the pyrolysis reactions 3 and 6 . Because neither condensation nor evaporation of gaseous species originating from heterogeneous reaction are considered in the frame of the present work, one is able to write:

$\left\langle\dot{w}_{i \neq H_{2} \mathrm{O}}\right\rangle=\left\langle\dot{w}_{i \neq \mathrm{H}_{2} \mathrm{O}}\right\rangle^{p y r}$

As it was mentioned above, for water the total production of vapour water is the sum of the production of gaseous water by pyrolysis reactions plus vapour originating from evaporation of liquid and bound water:

$\left\langle\dot{w}_{\mathrm{H}_{2} \mathrm{O}}\right\rangle=\left\langle\dot{w}_{\mathrm{H}_{2} \mathrm{O}}\right\rangle^{p y r}+\left\langle\dot{w}_{\mathrm{H}_{2} \mathrm{O}}\right\rangle^{\text {vap }}$

Where the vaporisation term is linked to the production terms encountered in (10) and (14) by:

$\left\langle\dot{w}_{H_{2} O}\right\rangle^{v a p}=-\left\langle\dot{w}_{l}\right\rangle-\left\langle\dot{w}_{b}\right\rangle$

Energy conservation. The last balance equation that can be written is the conservation of energy. The average internal energy for the volume under consideration is computed as a sum of the average internal energy of the different materials held within this volume. No interaction between them is considered in this work.

It can be also pointed out that the reference term implied in the estimation of these internal energies has been assigned to zero at the reference temperature and in their reference state at this temperature.
Finally, the heat of pyrolysis of organic material reaction $\Delta h_{O M}$ needs to be included as a source term, and so are the heat of vaporisation $\Delta h_{v a p}$ (and extra heat of desorption when bound water is considered) and of the heat of pyrolysis of the intermediate $\Delta h_{\text {int }}$ to take into account the net production of energy by these phenomena.

\section{Algebraic equations}

As it was stated in section 3.3.1, the complete knowledge of the system requires $\mathrm{NS}+10$ variables to be computed. However, given the fact that the choice has been made to sum equations for conservation of liquid and bound water we have not only added an extra variable $\left(\left\langle\rho_{l}\right\rangle+\left\langle\rho_{\boldsymbol{b}}\right\rangle\right)$, but also been able to derive only NS $+\mathbf{6}$ balance. Hence 5 equations are missing to complete the model. Moreover, in the previous equations, we were unable to assign a relation for the estimation of the rate of vaporisation of the sum of liquid and bound water $\left(\left\langle\dot{w}_{\mathrm{H}_{2} \mathrm{O}}\right\rangle^{\text {vap }}\right)$. This is the reason why we have extended the set of variables to be computed with this last one, and that 6 equations are now required to complete the model.

Ideal gas law. Pyrolysis is expected to be carried at atmospheric pressure at the external and at the surface of the basket. Of course the pressure within the sample should be higher than this value, because as liquid water is evaporated or as gases are produced by heterogeneous reactions, overpressure may arise. However we expect that the value of these overpressures should not exceed a value where the ideal gas law could not apply. This is the reason why one is able to write:

$\left\langle P_{g}\right\rangle^{g}=\frac{\left\langle\rho_{g}\right\rangle^{g} R\langle T\rangle}{M_{g}}$

where $M_{g}$ represents the local molar weight of the gas and is computed according to its local composition:

$\frac{1}{M_{g}}=\frac{1}{\left\langle\rho_{g}\right\rangle^{g}} \sum_{i=1}^{N S} \frac{\left\langle\rho_{i}\right\rangle^{g}}{M_{i}}$

In this last expression, $M_{i}$ is the molar weight of species I, and NS is the total number of chemical species 
Normalisation of gaseous composition. This equation provides a relation between intrinsic average mass concentration of gaseous species within the volume under consideration and the average total mass concentration of this gas:

$\left\langle\rho_{g}\right\rangle^{g}=\sum_{i=1}^{N S}\left\langle\rho_{i}\right\rangle^{g}$

Characterisation of water. As it was pointed above, Equation (19) enables us to compute $\left\langle\rho_{l}\right\rangle+\left\langle\rho_{b}\right\rangle$. However, and as it will be shown later, values for both $\left\langle\rho_{l}\right\rangle$ and $\left\langle\rho_{b}\right\rangle$ are required. This is the reason why we firstly introduce the total moisture content by:

$M=\frac{\left\langle\rho_{l}\right\rangle+\left\langle\rho_{b}\right\rangle}{\left\langle\rho_{S D}\right\rangle}$

Then given the Moisture content at fiber saturation point, $M_{f s p}$, which is an intrinsic property of the material that could be modified as the thermal treatment proceeds, one is able to compute the required variables as:

$\left\langle\rho_{b}\right\rangle=\left\langle\rho_{S D}\right\rangle \min \left(M_{f s p}, M\right)$

$\left\langle\rho_{l}\right\rangle=\left\langle\rho_{S D}\right\rangle \max \left(M-M_{f s p}, 0\right)$

Overall volume conservation. By nature, the theory of volume averaging requires that:

$\varepsilon_{g}+\varepsilon_{O M}+\varepsilon_{I N O}+\varepsilon_{C}+\varepsilon_{l}+\varepsilon_{b}+\varepsilon_{I N T}=1$

Substituting volume fractions of nongaseous phases by the ratio of their average mass concentration to their intrinsic mass concentration (which are known properties of the different materials) one is able to derive the next equation:

$\varepsilon_{g}=1-\left(\frac{\left\langle\rho_{l}\right\rangle}{\left\langle\rho_{l}\right\rangle^{l}}+\frac{\left\langle\rho_{b}\right\rangle}{\left\langle\rho_{b}\right\rangle^{b}}+\frac{\left\langle\rho_{\text {OM }}\right\rangle}{\left\langle\rho_{\text {OM }}\right\rangle^{\text {OM }}}+\frac{\left\langle\rho_{\text {INO }}\right\rangle}{\left\langle\rho_{\text {INO }}\right\rangle^{I N O}}+\frac{\left\langle\rho_{\text {Int }}\right\rangle}{\left\langle\rho_{\text {Int }}\right\rangle^{I n t}}+\frac{\left\langle\rho_{C}\right\rangle}{\left\langle\rho_{C}\right\rangle^{C}}\right)$

Liquid vapour equilibrium. The last equation that will be written relies on the assumption of equilibrium between liquid and/or bound water with vapour water. Indeed, this assumption leads to:

$\left\langle\rho_{\mathrm{H}_{2} \mathrm{O}}\right\rangle^{g}=\frac{a_{w} P_{\text {sat }} M_{\mathrm{H}_{2} \mathrm{O}}}{R\langle T\rangle}$

where $P_{\text {sat }}$ stands for the vapour pressure of pure water (estimated as a function of average local temperature, see below) and $\alpha_{w}$ represents the activity of bound or liquid water, and is equal to one if liquid water is present in the volume under consideration and equal to a function of temperature and bound water content if no more liquid water exists (see below).

\section{Physical and chemical data}

This section provides physical and chemical data that were used in the model.

Pyrolysis reaction. For this first try in using the model, the choice was made to use data from the reaction pathway of Di Blasi and Lanzetta, ${ }^{[30]}$ and the kinetic data obtained by C. Casajus ${ }^{[32]}$ in the case of Pine wood. Compared with our general reaction pathway, the one from Di Blasi and Lanzetta, ${ }^{[30]}$ is obtained considering reactions $1,2,4$ and 5 and neglecting reactions 3 and 6 and assuming that reactions 1 and 4 lead to the production of both permanent and condensable gases.

$n_{1}=n_{2}=n_{4}=n_{5}=1$
$k_{i}=A_{i} \exp \left(\frac{-E a_{i}}{R\langle T\rangle}\right)$

$E a_{1}=86936 \mathrm{~J} \mathrm{~mol}^{-1}, E a_{2}=87470 \mathrm{~J} \mathrm{~mol}^{-1}$,

$E a_{4}=111514 \mathrm{~J} \mathrm{~mol}^{-1}, E a_{5}=84238 \mathrm{~J} \mathrm{~mol}^{-1}$

$A_{1}=1.7 \times 10^{6} \mathrm{~min}^{-1}, A_{2}=41.5 \times 10^{4} \mathrm{~min}^{-1}$,

$A_{4}=59.1 \times 10^{1} \mathrm{~min}^{-1}, A_{2}=32.3 \times 10^{5} \mathrm{~min}^{-1}$

Capillary pressure. The work of Perre et al. ${ }^{[34]}$ was used to obtain the value of the capillary pressure as a function of temperature and total moisture content (defined in (35)).

$P_{C}=1.364 \times 10^{5} .(128-0.185 \times\langle T\rangle) \times 10^{-3} \times\left(M+1.2 \times 10^{-4}\right)^{-0.63}$

Heat of evaporation of water. Dealing with the heat of evaporation of water, a difference has to be made between liquid and bound water. Indeed, in the case of pure water no extra energy is required for desorption while it is the case for bound water. Hence:

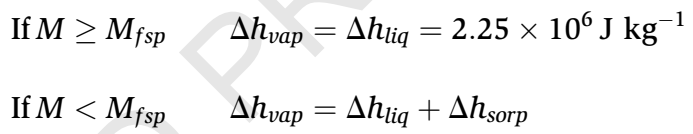

In this work, the heat of desorption has been computed according to the work of Stanish et al. ${ }^{[35]}$ :

$\Delta h_{\text {sorp }}=0.4 \Delta h_{\text {liq }}\left(1-\frac{\left\langle\rho_{b}\right\rangle}{\left\langle\rho_{S D}\right\rangle M_{f s p}}\right)^{2}$

Effective thermal conductivity. Because slow pyrolysis is mainly driven by heat transfer within the material, the estimation of effective thermal conductivity is one of the key parameter of the model. In his work, Gronli ${ }^{[25]}$ states that according to literature, the effective thermal conductivity of the material should be the sum of several terms, in order to include all possible transfers within each material (49).

$k_{e f f}=\varepsilon_{g} k_{g}+\left(\varepsilon_{b}+\varepsilon_{l}\right) k_{l}+\eta k_{S D}+(1-\eta) k_{C}+k_{r a d}$

In the above equation, $k_{g}$ (respectively $k_{l}, k_{S D}$, and $k_{c}$ ) stands for the intrinsic thermal conductivity of the gas (respectively, liquid, dry solid and char), $k_{\text {rad }}$ is the contribution of radiation to heat transfer within the medium, and $\eta$ represents the interpolation factor computed as:

$\eta=\frac{\left\langle\rho_{O M}\right\rangle+\left\langle\rho_{I N O}\right\rangle+\left\langle\rho_{\text {Int }}\right\rangle}{\left\langle\rho_{S D}\right\rangle}$

With the following expression for "radiation" thermal conductivity ${ }^{[25]}$

$k_{\text {rad }}=4 \frac{\varepsilon_{g}}{\left(1-\varepsilon_{g}\right)} \sigma_{r a d} \varepsilon_{r a d}\left(\eta d_{r a d, S D}+(1-\eta) d_{r a d, C}\right)\langle T\rangle^{3}$ 
Where $\sigma_{r a d}$ is the Stefan Boltzmann constant, $\varepsilon_{\text {rad }}$ is the emissivity of the material while $d_{r a d, \mathrm{SD}}$ (respectively $d_{\text {rad,c }}$ ) stands for the pore diameter of the dry solid (respectively the char).

Activity of water. According to the work of Gronli ${ }^{[25]}$ the following expression has been used to compute the activity of liquid water as a function of the temperature of the medium and of the state of liquid water (free or bound)

If $M \geq M_{f s p} \quad a_{w}=1$

If $M<M_{f s p} \quad a_{w}=1-\left(1-\frac{\left\langle\rho_{b}\right\rangle}{\left\langle\rho_{S D}\right\rangle M_{f s p}}\right)^{6.453 \times 10^{-3}\langle T\rangle}$

Relative permeabilities. According to the work of Perre and Turner, ${ }^{[34]}$ the following expressions have been used to compute the relative permeabilities of the gaseous and the liquid phases (where the properties of the sample have been assumed to the one of a longitudinal wood sample):

$K_{g}^{r}=1+(4 S-5) S^{4}$

$K_{l}^{r}=S^{8}$

In the previous equations, $S$ stands for the saturation of the medium and is defined as:

$S=\frac{M}{M_{s a t}-M_{f s p}}$

In Equation (56), $M_{\text {sat }}$ stands for the water content at saturation (ie in case the whole pores would be filled with liquid water). For our purposes, it has been evaluated by:

$M_{s a t}=\left\langle\rho_{l}\right\rangle^{l}\left(\frac{1}{\left\langle\rho_{S D}\right\rangle}-\frac{1}{\left\langle\rho_{I N O}\right\rangle^{I N O}}-\frac{1}{\left\langle\rho_{\text {Int }}\right\rangle^{\text {Int }}}-\frac{1}{\left\langle\rho_{O M}\right\rangle^{O M}}\right)$

Other properties. The other numerical values of the properties that were used in the model are summed up in Table 2

\section{Boundary conditions}

As the model involves partial differential equations, depending upon space and time, some initial and boundary conditions are required in order to make the solution a unique one. Hence, the next paragraphs provide information on these conditions.

Initial conditions. Dealing with the initial conditions, it is here after stated that at the initial time, the medium is at rest, with composition given by its proximate analysis. More precisely, this means that the initial average concentrations of bound and liquid water are computed from the initial moisture content of the sample while the initial average concentrations of organic and inorganic material are computed from the ash and organic content of the sample. The composition of the gas that the pores hold and that are not filled with water is supposed to be composed of air and vapour water. The amount of vapour water in air is computed according to equilibrium assumption between liquid and vapour water at the initial temperature of the medium. Of course, these compositions are supposed to be uniform all over the sample.

Radial boundary conditions. Two boundary conditions have to be written for each state variables involved in partial differential equations. More precisely, it has been chosen here to write one boundary condition on the axis of the basket (symmetry condition) and one on the wall of the basket (no mass transfer but heat transfer). The conditions can be written as:

$\underline{\text { Axis }}$

$$
\begin{aligned}
& \left.\frac{\partial\langle T\rangle}{\partial r}\right|_{t, z, r=0}=0 \\
& \left.\frac{\partial\left(\left\langle\rho_{l}\right\rangle+\left\langle\rho_{b}\right\rangle\right)}{\partial r}\right|_{t, z, r=0}=0 \\
& \left.\frac{\partial\left\langle\rho_{i}\right\rangle^{g}}{\partial r}\right|_{t, z, r=0}=0
\end{aligned}
$$

\section{Wall}

$$
\begin{aligned}
& -\left.\lambda_{\text {eff }} \frac{\partial\langle T\rangle}{\partial r}\right|_{t, z, r=R}-\left.c_{p, l}\langle T\rangle \frac{\left\langle\rho_{l}\right\rangle^{l} K_{l} K_{l}^{r}}{\mu_{l}} \frac{\partial\left\langle P_{l}\right\rangle^{g}}{\partial r}\right|_{t, z, r=R}-\left.c_{p, l}\langle T\rangle D_{b} \frac{\partial\left\langle\rho_{b}\right\rangle}{\partial r}\right|_{t, z, r=R} \\
& -\left.c_{p, g}\langle T\rangle \frac{\left\langle\rho_{g}\right\rangle^{g} K_{g} K_{g}^{r}}{\mu_{g}} \frac{\partial\left\langle P_{g}\right\rangle^{g}}{\partial r}\right|_{t, z, r=R}-\left.\sum_{i=1}^{N S} c_{p, g}\langle T\rangle\left\langle\rho_{g}\right\rangle^{g} D_{i, g} \frac{\partial}{\partial r}\left(\frac{\left\langle\rho_{i, g}\right\rangle^{g}}{\left\langle\rho_{g}\right\rangle^{g}}\right)\right|_{t, z, r=R} \\
& =h_{T, s i d}\left(\langle T\rangle_{t, z, r=R}-T_{\infty}\right)+\varepsilon_{r a d} \sigma_{r a d}\left(\langle T\rangle_{t, z, r=R}^{4}-T_{\infty}^{4}\right) \\
& -\left.\left\langle\rho_{l}\right\rangle^{l_{l}} \frac{K_{l} K_{l}^{r}}{\mu_{l}} \frac{\partial\left\langle P_{l}\right\rangle^{l}}{\partial r}\right|_{t, z, r=R}-\left.D_{b} \frac{\partial\left\langle\rho_{b}\right\rangle}{\partial r}\right|_{t, z, r=R}=0 \\
& -\left.\left\langle\rho_{i, g}\right\rangle^{g} \frac{K_{g} K_{g}^{r}}{\mu_{g}} \frac{\partial\left\langle P_{g}\right\rangle^{g}}{\partial r}\right|_{t, z, r=R}-\left\langle\rho_{g}\right\rangle^{g} D_{g} \frac{\partial}{\partial r}\left(\left.\frac{\left\langle\rho_{i, g}\right\rangle^{g}}{\left\langle\rho_{g}\right\rangle^{g}}\right|_{t, z, r=R}=0\right.
\end{aligned}
$$

Axial boundary conditions. Once again, because of the nature of the partial differential equations involving the state variables, two boundary conditions are required for $\langle T\rangle,\left\langle\rho_{l}\right\rangle+\left\langle\rho_{b}\right\rangle$ and $\left\langle\rho_{i}\right\rangle^{\mathrm{g}}$, both at the bottom and at the top of the basket. At the bottom of the basket, flux conditions are imposed because nitrogen is fed to the sample at this position with known temperature and gas composition. Dealing with liquid and bound water, it is hereafter stated that there is no flux of this material at this location. This is also the case at the top of the basket. At this position, a flux condition is also imposed for temperature, whereas a condition of no diffusion is associated to gaseous species. This condition of no diffusion is set for all species except nitrogen for which the condition is replaced by a gaseous pressure condition which is fixed to the atmospheric value.

Bottom

$$
\begin{aligned}
& -\left.\lambda_{\text {eff }} \frac{\partial\langle T\rangle}{\partial z}\right|_{t, r, z=0}-\left.c_{p, l}\langle T\rangle \frac{\left\langle\rho_{l}\right\rangle^{l} K_{l} K_{l}^{r}}{\mu_{l}} \frac{\partial\left\langle P_{l}\right\rangle^{g}}{\partial z}\right|_{t, r, z=0}-\left.c_{p, l}\langle T\rangle D_{b} \frac{\partial\left\langle\rho_{b}\right\rangle}{\partial z}\right|_{t, r, z=0} \\
& -\left.c_{p, g}\langle T\rangle \frac{\left\langle\rho_{g}\right\rangle^{g} K_{g} K_{g}^{r}}{\mu_{g}} \frac{\partial\left\langle P_{g}\right\rangle^{g}}{\partial z}\right|_{t, r, z=0}-\left.\sum_{i=1}^{N S} c_{p, g}\langle T\rangle\left\langle\rho_{g}\right\rangle^{g} D_{i, g} \frac{\partial}{\partial z}\left(\frac{\left\langle\rho_{i, g}\right\rangle^{g}}{\left\langle\rho_{g}\right\rangle^{g}}\right)\right|_{t, r, z=0}= \\
& -\varepsilon_{\text {rad }} \sigma_{r a d}\left(\langle T\rangle_{t, r, z=0}^{4}-T_{\infty}^{4}\right)+c_{p, g} \rho_{g, i n c} U_{\text {inc }} T_{\text {inc }}
\end{aligned}
$$




$$
\begin{aligned}
& -\left.\left\langle\rho_{l}\right\rangle^{l_{l} K_{l} K_{l}^{r}} \frac{\partial\left\langle P_{l}\right\rangle^{l}}{\partial z}\right|_{t, r, z=0}-\left.D_{b} \frac{\partial\left\langle\rho_{b}\right\rangle}{\partial z}\right|_{t, r, z=0}=0 \\
& -\left.\left\langle\rho_{i, g}\right\rangle^{g} \frac{K_{g} K_{g}^{r}}{\mu_{g}} \frac{\partial\left\langle P_{g}\right\rangle^{g}}{\partial z}\right|_{t, r, z=0}-\left.\left\langle\rho_{g}\right\rangle^{g} D_{g} \frac{\partial}{\partial z}\left(\frac{\left\langle\rho_{i, g}\right\rangle^{g}}{\left\langle\rho_{g}\right\rangle^{g}}\right)\right|_{t, r, z=0}=\rho_{g, i n c} U_{i n c} Y_{i, i n c}
\end{aligned}
$$

In the above expressions, $\rho_{g}$,inc $, U_{i n c}, T_{i n c}$ and $Y_{i, i n c}$ respectively stands for the density of the gas fed to the sample, its velocity, temperature and composition (in terms of mass fraction).

Top

$$
\begin{aligned}
& -\left.\lambda_{e f f} \frac{\partial\langle T\rangle}{\partial z}\right|_{t, r, z=H}-\left.c_{p, l}\langle T\rangle \frac{\left\langle\rho_{l}\right\rangle^{l} K_{l} K_{l}^{r}}{\mu_{l}} \frac{\partial\left\langle P_{l}\right\rangle^{g}}{\partial z}\right|_{t, r, z=H}-\left.c_{p, l}\langle T\rangle D_{b} \frac{\partial\left\langle\rho_{b}\right\rangle}{\partial z}\right|_{t, r, z=H} \\
& -\left.\sum_{i=1}^{N S} c_{p, g}\langle T\rangle\left\langle\rho_{g}\right\rangle^{g} \frac{\partial}{\partial z}\left(\frac{\left\langle\rho_{i, g}\right\rangle^{g}}{\left\langle\rho_{g}\right\rangle^{g}}\right)\right|_{t, r, z=H}=\varepsilon_{r a d} \sigma_{r a d}\left(\langle T\rangle_{t, r, z=H}^{4}-T_{\infty}^{4}\right)
\end{aligned}
$$

$-\left.\left\langle\rho_{l}\right\rangle \frac{K_{l} K_{l}^{r}}{\mu_{l}} \frac{\partial\left\langle P_{l}\right\rangle^{l}}{\partial z}\right|_{t, r, z=H}-\left.D_{b} \frac{\partial\left\langle\rho_{b}\right\rangle}{\partial z}\right|_{t, r, z=H}=0$

$-\left.\left\langle\rho_{g}\right\rangle^{g} D_{g} \frac{\partial}{\partial z}\left(\frac{\left\langle\rho_{i, g}\right\rangle^{g}}{\left\langle\rho_{g}\right\rangle^{g}}\right)\right|_{t, r, z=H}=0 \quad i \neq N_{2}$

$\left.\left\langle P_{g}\right\rangle^{g}\right|_{t, r, z=H}=P_{0}$

\section{Overall model}

Table 1 gives a sum up of the state variables of the model and the associated equations. The model is thus composed of NS +2 Partial Differential Equations (PDE's), 4 Ordinary Differential Equations (ODE's), and 7 Algebraic Equations (AE), where NS stands for the total number of gaseous species taken into account in the model.

\section{SOLVING}

As it was mentioned in the preceding section, the overall model is composed of PDE's, ODE's and AE. The first step that was used to solve it was firstly to convert PDE's into ODE's using the method of lines. More precisely, this method relies on a spatial discretization of the PDE's in order to convert them into ODE's on a computational and Cartesian grid. In our case, a vertex centered finite volumes method was used. ${ }^{[36]}$

Once this step has been done, the resulting model is a set of ordinary differential (with respect to time) and algebraic equations of the form:

$M(t, y) \frac{d y}{d t}=f(t, y)$

Where $y$ is the vector containing the state variables (which now includes the variables at the nodes of the computational grid), $M$ is the mass matrix, which is singular because of the occurrence of algebraic equations within the overall model. This system has been solved in our case using a DASSL Method, ${ }^{[37,38]}$ and more precisely the DisCO Library ${ }^{[39]}$ was used for our integration purpose. It has to be noticed here that, in order to reduce the computational time and to improve convergence rate, the Jacobian of $f$ and M mass matrix involved in the solving and more precisely on the corrector step, where computed analytically.

\section{RESULTS}

This part of the paper is devoted to the discussion of the results obtained using the model. In a first part, some details on the sample being analyzed as well as on the pyrolysis conditions used will be given. Then, some insights on the numerical data will be provided to the reader. Before a deeper analyze of the results provided by the model, a validation step will be done. It allows comparing the experimental mass loss of the sample with the one computed by the model.

\section{Sample Under Consideration and Operating Conditions}

\begin{tabular}{|c|c|c|c|c|}
\hline \multicolumn{2}{|l|}{ Variables } & \multicolumn{3}{|l|}{ Equations } \\
\hline Name & Symbol & Name & Number & Type \\
\hline Average temperature & $\langle T\rangle$ & Energy balance & $(31)$ & PDE \\
\hline Average mass concentration of organic material. & $\left\langle\rho_{\mathrm{OM}}\right\rangle$ & Organic material mass balance & $(5)$ & ODE \\
\hline Average mass concentration of Intermediate & $\left\langle\rho_{\text {Int }}\right\rangle$ & Intermediate mass balance & (7) & ODE \\
\hline Average mass concentration of inorganic material & $\left\langle\rho_{I N O}\right\rangle$ & Inorganic material mass balance & (9) & ODE \\
\hline Average mass concentration of char & $\left\langle\rho_{C}\right\rangle$ & Char mass balance & (10) & ODE \\
\hline Average mass concentration of bound plus liquid water & $\left\langle\rho_{l}\right\rangle+\left\langle\rho_{b}\right\rangle$ & Sum of liquid and bound water mass balances & (19) & $\mathrm{PDE}$ \\
\hline Intrinsic average mass fraction of species (NS Variables) & $\left\langle\rho_{i}\right\rangle^{g}$ & Species mass balance (NS equations) & (20) & PDE \\
\hline Intrinsic average pressure of the gaseous phase & $\left\langle P_{g}\right\rangle^{g}$ & Ideal gas law & (32) & $\mathrm{AE}$ \\
\hline Intrinsic average total mass concentration of gas & $\left\langle\rho_{g}\right\rangle^{g}$ & Normalisation equation & (34) & $\mathrm{AE}$ \\
\hline Average mass conservation of bound water & $\left\langle\rho_{b}\right\rangle$ & Characterisation of water & (36) & $\mathrm{AE}$ \\
\hline Average mass conservation of liquid water & $\left\langle\rho_{l}\right\rangle$ & Characterisation of water & (37) & $\mathrm{AE}$ \\
\hline Fraction of volume occupied by gas & $\varepsilon_{g}$ & Overall volume conservation & (39) & $\mathrm{AE}$ \\
\hline Interpolation factor & $\eta$ & Definition & $(50)$ & $\mathrm{AE}$ \\
\hline Rate of vaporisation of liquid and bound water & $\left\langle\dot{w}_{\mathrm{H}_{2} \mathrm{O}}\right\rangle^{v a p}$ & Equilibrium equation & (40) & $\mathrm{AE}$ \\
\hline
\end{tabular}

The sample under consideration is pine wood, previously shred with an average diameter of $1 \mathrm{~mm}$. The analysis of the

Table 1. Summary of variables (and associated equations) computed in the model 
Table 2. Values of the physical parameters used in the model

\begin{tabular}{|c|c|c|c|}
\hline Name & Symbol & Value & Units \\
\hline Heat capacityof gas & $c_{p, g}$ & 1100 & $\mathrm{Jkg}^{-1}$ \\
\hline Heat capacity of liquid water & $c_{p, l}$ & 4180 & $\mathrm{Jg}^{-1}$ \\
\hline Heat capacity of organic material & $c_{p, \mathrm{OM}}$ & 900 & $\mathrm{~kg}^{-1}$ \\
\hline Heat capacity of inorganic material & $c_{p, I N O}$ & 950 & $\mathrm{jg}^{-1}$ \\
\hline Heat capacity of Intermediate & $c_{p, \ln t}$ & 900 & $\mathrm{jg}^{-1}$ \\
\hline Heat capacity of char & $c_{p, C}$ & 700 & $\mathrm{Jg}^{-1}$ \\
\hline Intrinsic gaseous permeability of dry solid & $K_{g, S D}$ & $10^{-12}$ & $\mathrm{~m}^{2}$ \\
\hline Intrinsic gaseous permeability of char & $K_{g, C}$ & $10^{-9}$ & $\mathrm{~m}^{2}$ \\
\hline Dynamic viscosity of liquid water & $\mu_{1}$ & $5 \times 10^{-4}$ & Pas \\
\hline Dynamic viscosity of gas & $\mu_{g}$ & $3 \times 10^{-5}$ & Pas \\
\hline Diffusion coefficient of species I within the gaseous mixture & $D_{i}$ & $2.5 \times 10^{-5}$ & $m^{2} s^{-1}$ \\
\hline Intrinsic thermal conductivity of the gas & $k_{g}$ & 0.025 & $\mathrm{~W} \mathrm{~m}^{-1} \mathrm{~K}^{-1}$ \\
\hline Intrinsic thermal conductivity of the liquid & $k_{l}$ & 0.658 & $\mathrm{Wm}^{-1} \mathrm{~K}^{-1}$ \\
\hline Intrinsic thermal conductivity of the dry solid & $k_{S D}$ & 0.15 & $\mathrm{~W} \mathrm{~m}^{-1} \mathrm{~K}^{-1}$ \\
\hline Intrinsic thermal conductivity of the char & $k_{C}$ & 0.15 & $\mathrm{~W} \mathrm{~m}^{-1} \mathrm{~K}^{-1}$ \\
\hline Pore diameter of the dry solid & $d_{\text {rad, }, S D}$ & $50 \times 10^{-6}$ & $\mathrm{~m}$ \\
\hline Pore diameter of the char & $d_{\text {rad, } \mathrm{c}}$ & $100 \times 10^{-6}$ & $\mathrm{~m}$ \\
\hline Emissivity of the medium & $\varepsilon_{\text {rad }}$ & 0.6 & \\
\hline
\end{tabular}

material is provided in Table 3 . The initial density of the material is $286.5 \mathrm{~kg} / \mathrm{m}^{3}$. The basket of the furnace (Bk 101) is loaded with $200 \mathrm{~g}$ of this material.

Once the furnace has been tightly closed, the first step is to feed the device with nitrogen with a volumetric flowrate of $15 \mathrm{Nl} / \mathrm{min}\left(2.5 \times 10^{-4} \mathrm{Nm}^{3} / \mathrm{s}\right)$. Hence, in the first six minutes of the experiment, nitrogen is progressively introduced in the system. Then the temperature of the furnace (as well as this of the incoming nitrogen) is raised to $110^{\circ} \mathrm{C}$ at a heating rate of $10^{\circ} \mathrm{C} / \mathrm{min}$. This temperature is then maintained for 60 minutes in order to dry the sample and in order to evacuate the remaining oxygen from the sample and the furnace. After the drying step, the temperature of the furnace and of the incoming nitrogen are raised to $285^{\circ} \mathrm{C}$ at a heating rate of $20^{\circ} \mathrm{C} / \mathrm{min}$ and these conditions are kept during 5 minutes. Then the power sent to the furnace and to the preheater is shut off and the sample cools. The evolution of the operating conditions are provided in Figure 5.

\section{Numerical Parameters}

In this study, the simulations were performed on a mesh composed of 14 nodes on both directions. Hence, the total number of nodes was 196 . Given the number of gaseous species

\begin{tabular}{lc}
\multicolumn{2}{c}{ Table 3. Analysis of the material under consideration } \\
\hline \multicolumn{2}{c}{ Composition of the pine used in the study } \\
\hline Proximate analysis (\% mass, raw) & 2.0 \\
Ash & 11.36 \\
Moisture & 86.64 \\
Organic matter & 51 \\
Ultimate analysis (\% mass, daf, XP CEN/TS 15104) & 6 \\
C & 42 \\
H & $<0.3$ \\
O (difference) & 11 \\
N & 53 \\
Molecular composition (\%mass, dry XP CEN/TS 15104) & 25 \\
Hemicellulose & 19.36 \\
Cellulose & \\
Lignin & \\
Lower heating value (MJ/kg, dry, XP CEN/TS 14918) & \\
&
\end{tabular}

taken into account in the model $(\mathrm{NS}=16)$, the total number (per node of the mesh) of ODE's is 22 while the number of AE is 7 . Hence the overall number of equations to be solved was 5684. In order to avoid the solving of a linear system involving a square matrix of $5684^{*} 5684$ elements in the corrector step, we have chosen an analytical and sparse matrix for both mass and jacobian matrices. Indeed, the overall convergence matrix was composed of solely 127712 non-zeros elements which is only $0.4 \%$ of the overall square matrix.

\section{Validation}

This paragraph is devoted to the validation of the model. A complete validation should include not only the comparison of the experimental and numerical mass loss of the sample, but also a comparison of both the production (as a function of time) of the condensable and permanent gases. However, in the first step of the study, such a comparison was not possible. Indeed, we were unable to find experimental data on the estimation of the composition of the gases released during the two steps of the

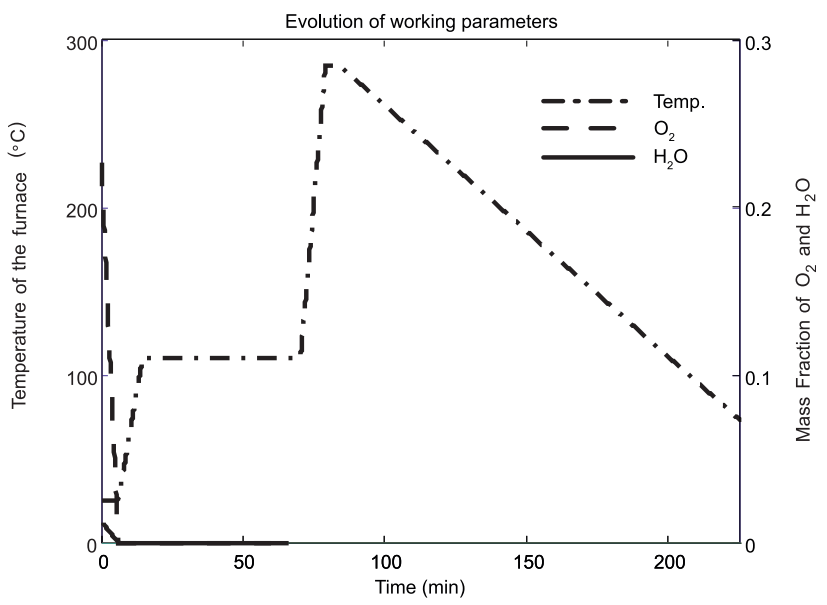

Figure 5. Evolution of the operating parameters of the device. Temperature of the furnace and of the incoming gas, mass composition of the incoming gas (in terms of $\mathrm{H}_{2} \mathrm{O}$ and $\mathrm{O}_{2}$ ). 


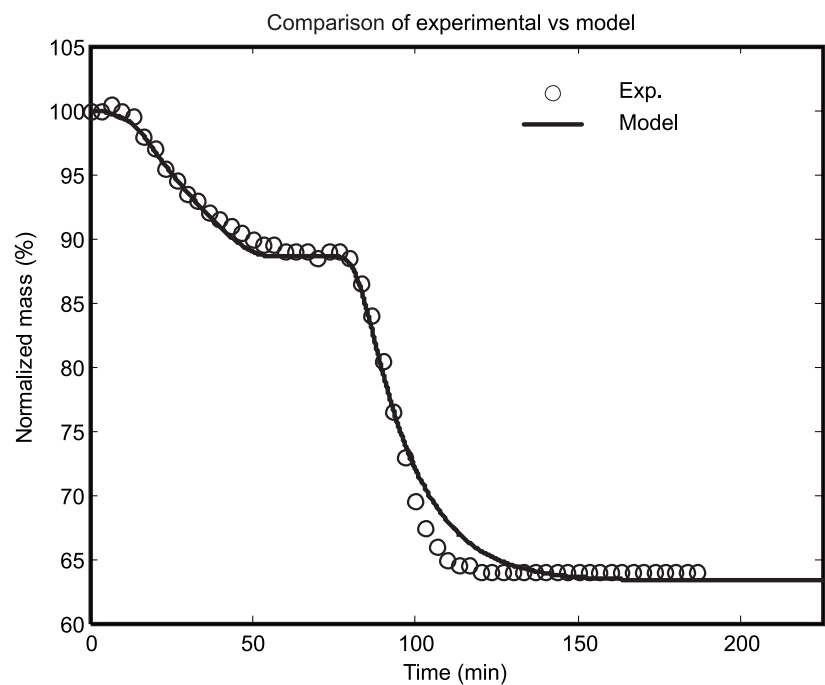

Figure 6. Mass loss of the sample (in terms of normalized mass). Comparison of experimental and numerical results. pyrolysis step. We are confident on the fact that using this model and the results obtained by the experimental device, will enable us to compute and propose this kind of data shortly. Hence, in this work, It was only possible to validate (partially) the model comparing the numerical and experimental mass loss and this is precisely the scope of the next paragraph.

Also, it would have been interesting to compare the numerical differential mass loss (derivative of mass loss as a function of time) with the experimental one. However, because of the precision of the balance $\mathrm{Wg} 101$, which is $1 \mathrm{~g}$, it was not possible to extract the information from the experimental study. We plan to change this balance for a one with a precision of $0.1 \mathrm{~g}$, what will allow us to make more accurate comparisons.

It would also have been interesting to compare internal temperature profile within the sample as a function of time. However, this measure strongly affects the measure of the mass of the sample. Hence we plan to make two experiments in the futur, the first one will allow the estimation of the mass loss (without measurement of internal temperature profile) while the second will allow the estimation of the internal temperature profile (without estimation of the mass loss).

Figure 6 shows the evolution of the mass of the sample (in terms of normalized mass) as a function of time for both experimental and numerical points of view. It can be seen that the numerical
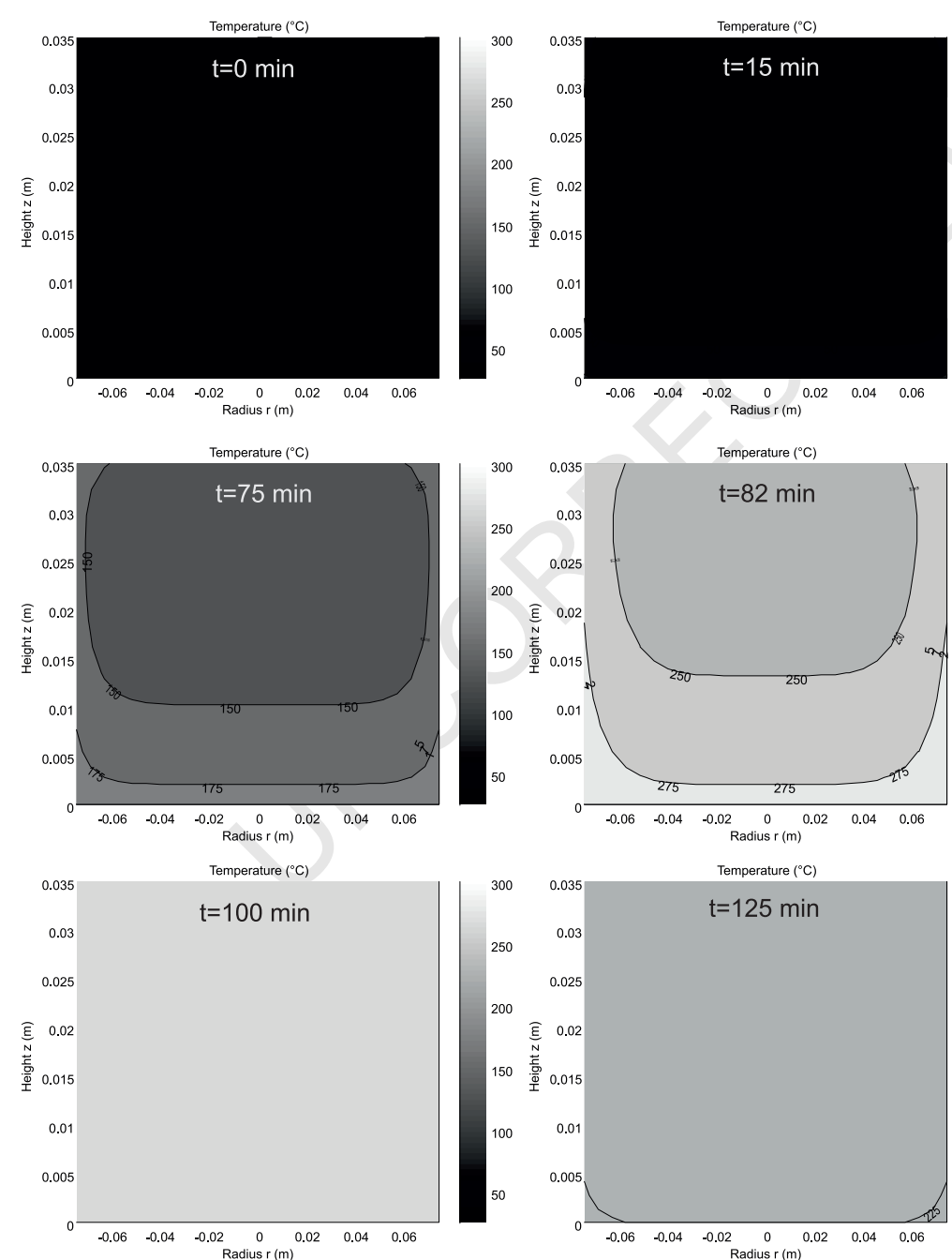
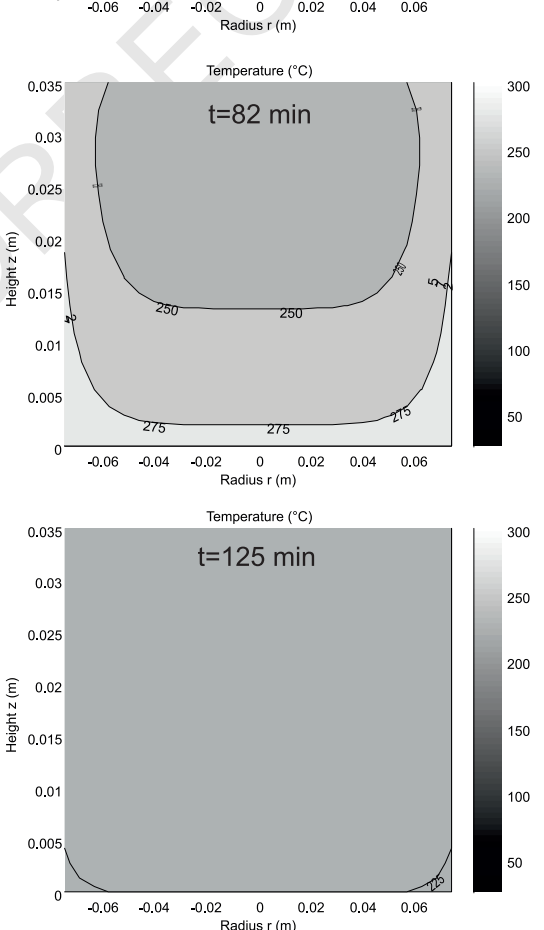
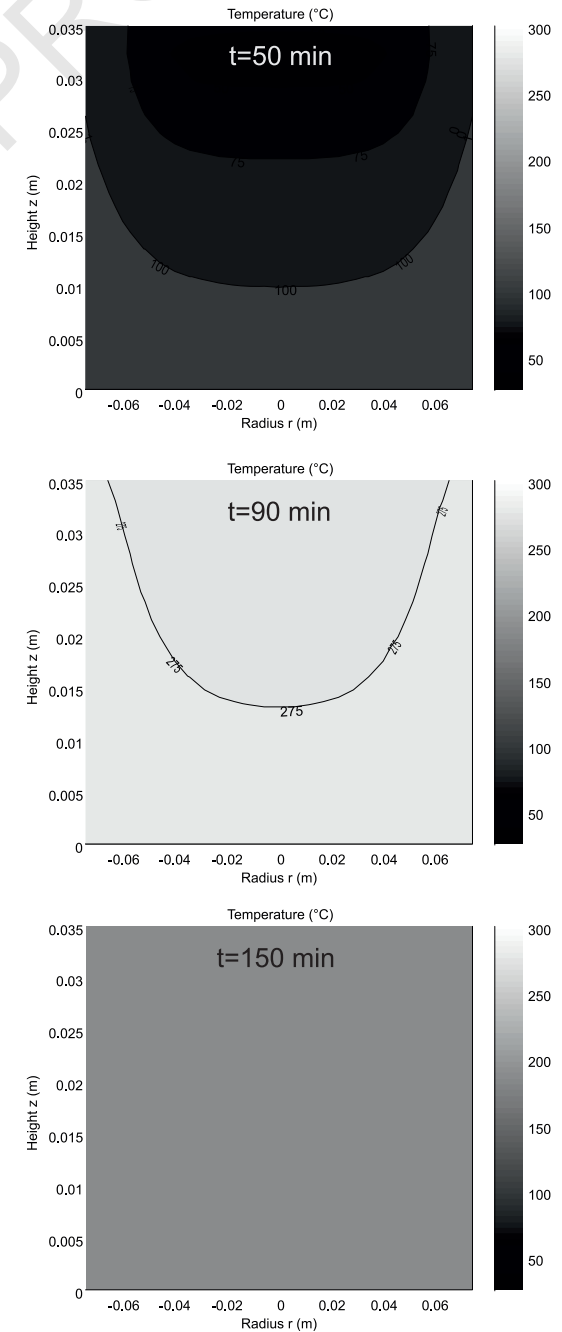

Figure 7. Evolution of the temperature of the material within the basket as a function of space and time. 
prediction for the drying step fits the experimental results quite well. Indeed, both the slope of the mass loss, and the corresponding characteristics times are quite the same. This emphasizes the fact that both equations and numerical data used in the model allow a good description of the processes occurring in the drying step. Dealing with the pyrolysis step, it can be said that the first step of this process is well represented, but it seems that its end is under estimated by the model. This could be associated to an overestimation of the prediction of the cooling of the sample (being itself associated to an overestimation of the internal thermal conductivity). Indeed, it would be necessary to have a parametric analysis of the different parameters of the model. However, this is not in the scope of the paper, and hereafter we consider that the comparison of the results is sufficient at the present time to show the ability of the model to describe the internal phenomena occurring in the sample.

\section{Analysis of the results}

Figures 7-10 give a $2 \mathrm{D}$ representation of the internal profiles of temperature, moisture content, organic material content and char content held in the basket Bk101 times $(0,15,50,75,82,90,125$ and $150 \mathrm{~min}$ ). The abscissa of each sub figure shows the radius of the basket, while the ordinate shows the height of the biomass held in this basket. Regarding Figure 8 It can firstly be seen that the drying step is quite finished 50 minutes after the starting of the experiment (the moisture content is almost zero within the whole basket), and that his drying step proceeds mainly from the bottom of the sample (where pure nitrogen is fed, and where the moisture content becomes 0 ate first, see Fig. 8 at $15 \mathrm{~min}$ )) and to a lower extent at the top of the basket where heat is directly brought by radiation to the sample (without the intermediate of walls) (at 15 minutes, the water content at the top of the basket is lower, $90 \%$, than at the heart of the basket where the moisture content is $100 \%)$. It can also be stated that this drying step operates at a drying front (leaving the dry material behind it) that progressively moves from the bottom to the top of the sample. It can also be seen that while the material contains moisture, its temperature does not reach the temperature of the furnace. More precisely, after 15 minutes (where the temperature of the furnace is $110^{\circ} \mathrm{C}$, see Fig. 5), the temperature inside the basket is almost $25^{\circ} \mathrm{C}$ (see Fig. 7) This is only once the material held in the basket is dried that its temperature is raised. Indeed after 50 minutes, the lower part of the sample has reached $110^{\circ} \mathrm{C}$ while the top (where there is still moisture) is still at a temperature of about $50^{\circ} \mathrm{C}$.

After 75 minutes, the sample can be considered to be perfectly dry (See Fig. 8, the moisture content is 0 at every location in the basket), and its temperature rises again (see Fig. 7, the temperature within the basket is raised up to $175^{\circ} \mathrm{C}$ ), but not as quickly as
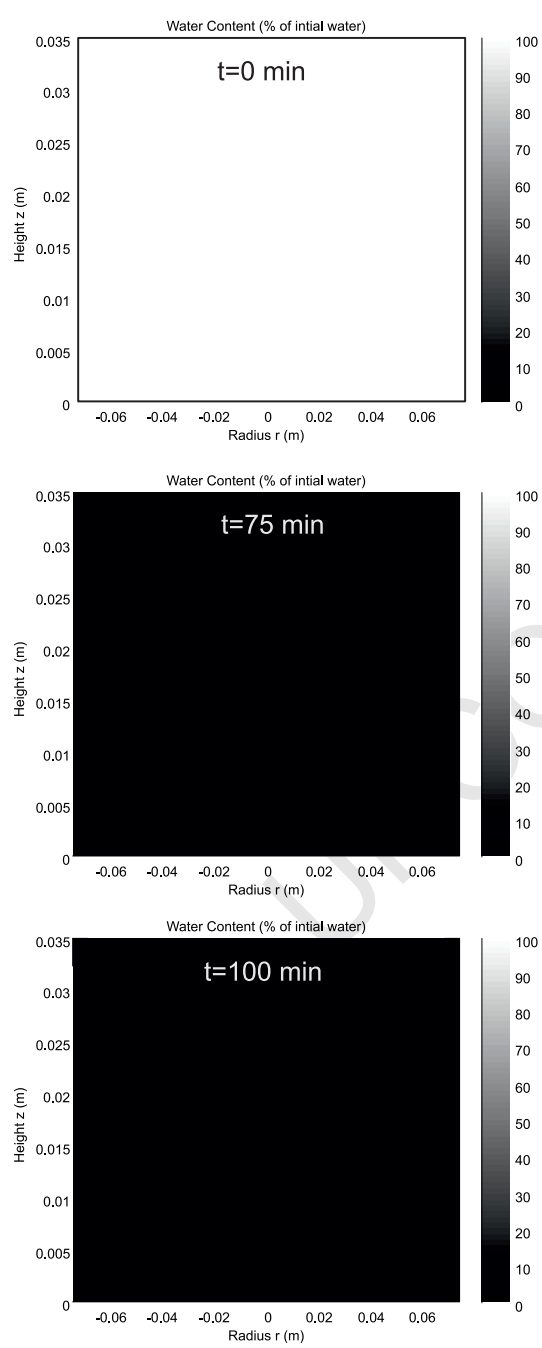
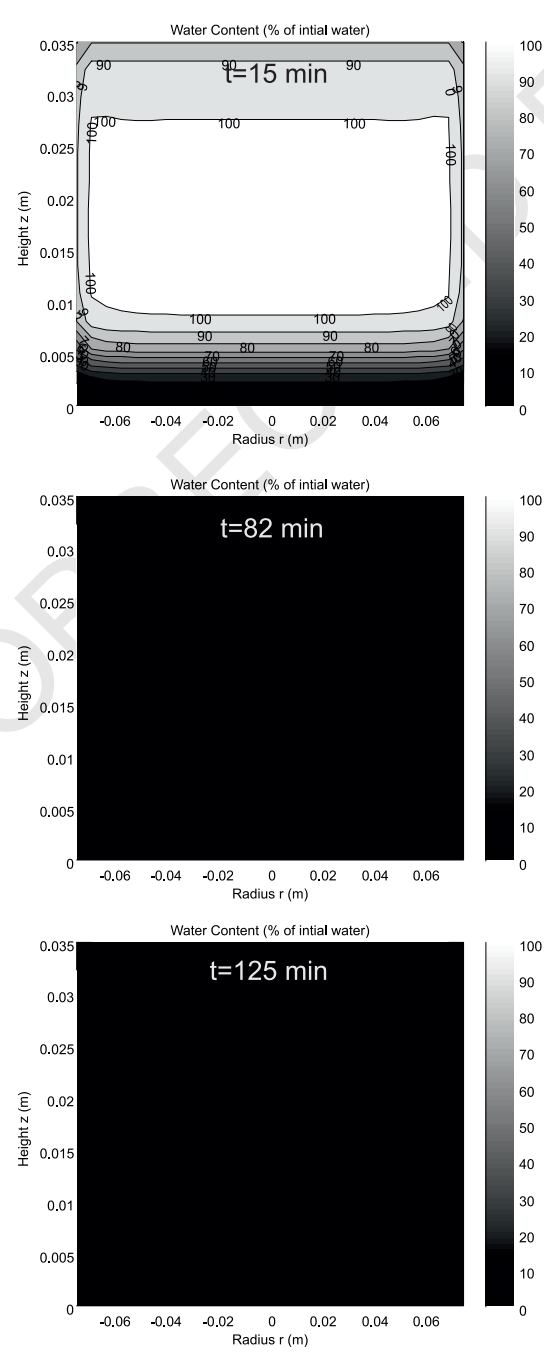
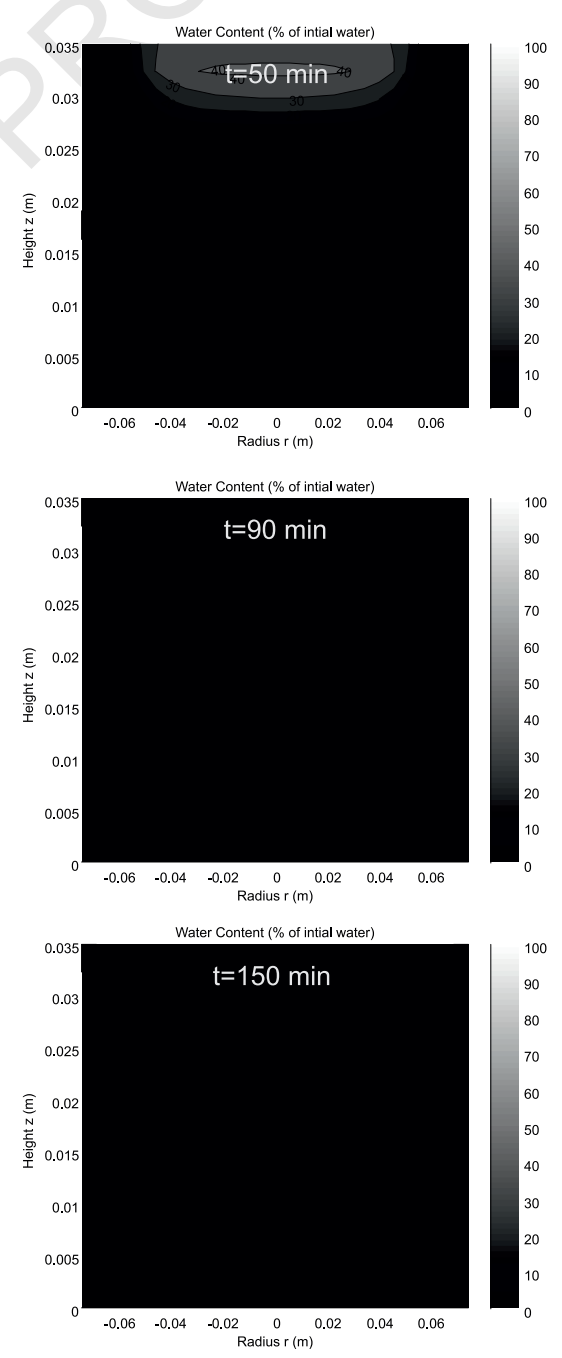

Figure 8. Evolution of the moisture content of the material within the basket as a function of space and time. 

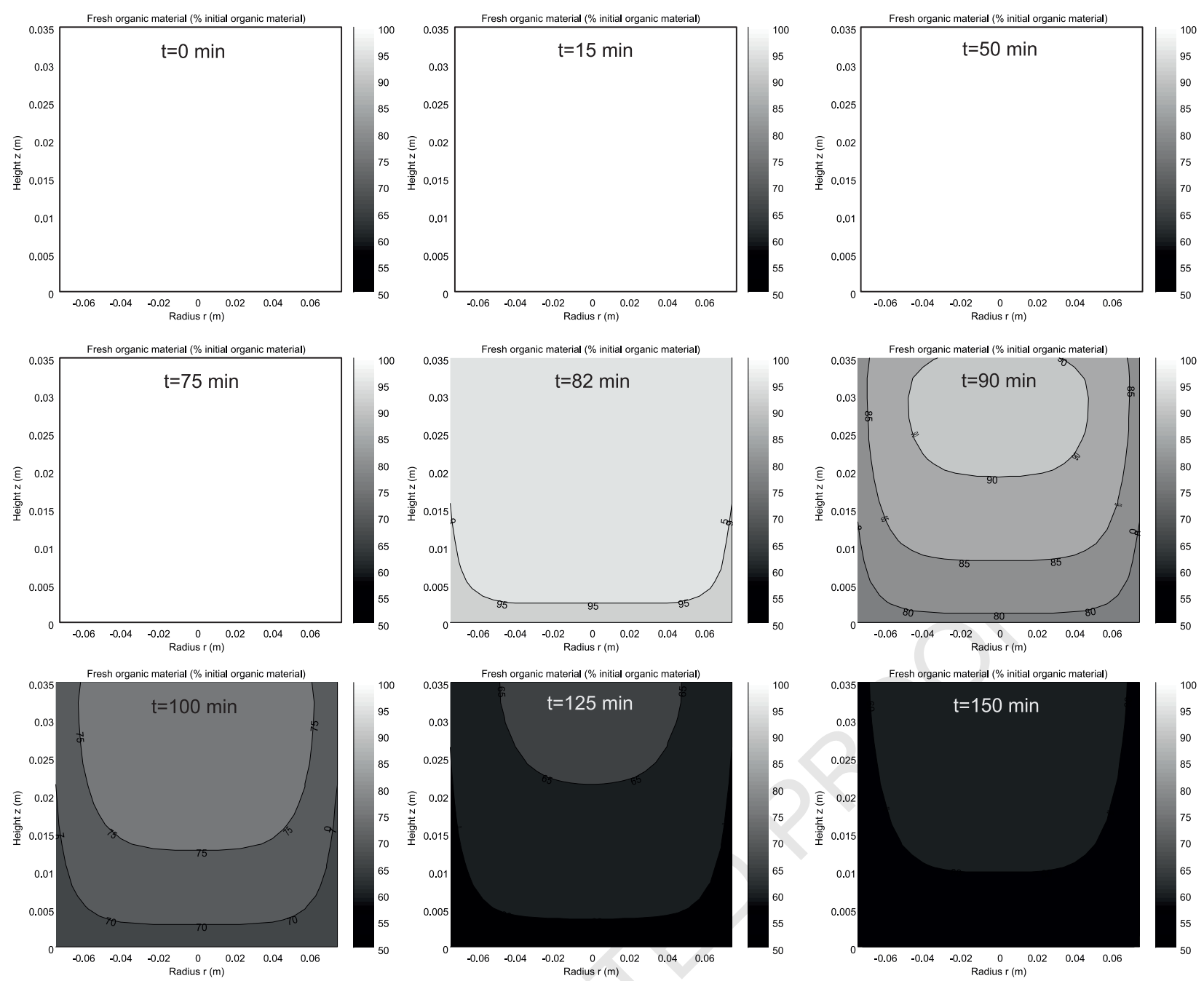

Figure 9. Evolution of the organic content (in terms of percentage of the initial organic content) of the material within the basket as a function of space and time.

the temperature of the furnace does. More precisely, after $82 \mathrm{~min}$ the maximal temperature is reached $\left(285^{\circ} \mathrm{C}\right.$, see Fig. 5) but the sample is still below this value (see Fig. 7, the maximal temperature in the basket is $275^{\circ} \mathrm{C}$ ) what shows the influence of heat transfer within it. However, it can be seen that, the pyrolysis has started because the organic content has diminished (see Fig. 9, at 82 minutes the maximal organic content is $95 \%$ ) while the char one has been raised (see on Fig. 10 after 82 minutes of processing, the colour sweeps form black to dark grey at the bottom ends of the sample). This reaction proceeds up to 125 minutes after the starting of the unit, even though the temperature of the furnace has begun to slightly decrease(Figs. 9 and 10 are modified from $t=100$ minutes to $\mathrm{t}=150$ minutes what proves that the organic content decreases during this time and simultaneously the char content increases). After 150 minutes, the pyrolysis process has stopped because of the low value of the local temperature. It can also be stated, that at the end of the process, approximately $40 \%$ of the organic material has been converted into char $(12 \%$ of the initial organic content) and gases, and that this degradation is quite homogeneous within the basket.

\section{CONCLUSION}

This paper has presented the development of a two dimensional and transient model for pyrolysis of biomass in a fixed bed. This model mainly relies on the theory of volume averaging in order to convert balance equations obtained at the phase scale in the heterogeneous medium under consideration into balance equations available on an elementary representative and continuous volume. Using this theory we were able to present the balance and algebraic equations that describe heat and mass transfers as well as reactions within the sample under consideration. This model has been applied to the pyrolysis of a sample of $200 \mathrm{~g}$ of pine wood in a high capacity thermobalance that has been developed in the laboratory. This device allows the measurement of the mass loss of the sample as well as the composition of the condensable and permanent gases released during the operation.

Comparison of the numerical mass loss of the sample with the numerical one showed good agreement, what allowed us to partially validate the model. Indeed, supplementary validation should be performed in particular in terms of internal temperature profile as well as differential mass loss and composition of the permanent and condensable gases leaving the device. This has not been done yet, but the partial validation that was obtained is a first step. Analysis of the results provided by the model gave us information on the pyrolysis operation within the device.

Once the extra validation will be performed, it will also be necessary to make a parametric study in order to check for the influence of the different parameters used in the model. Then, 

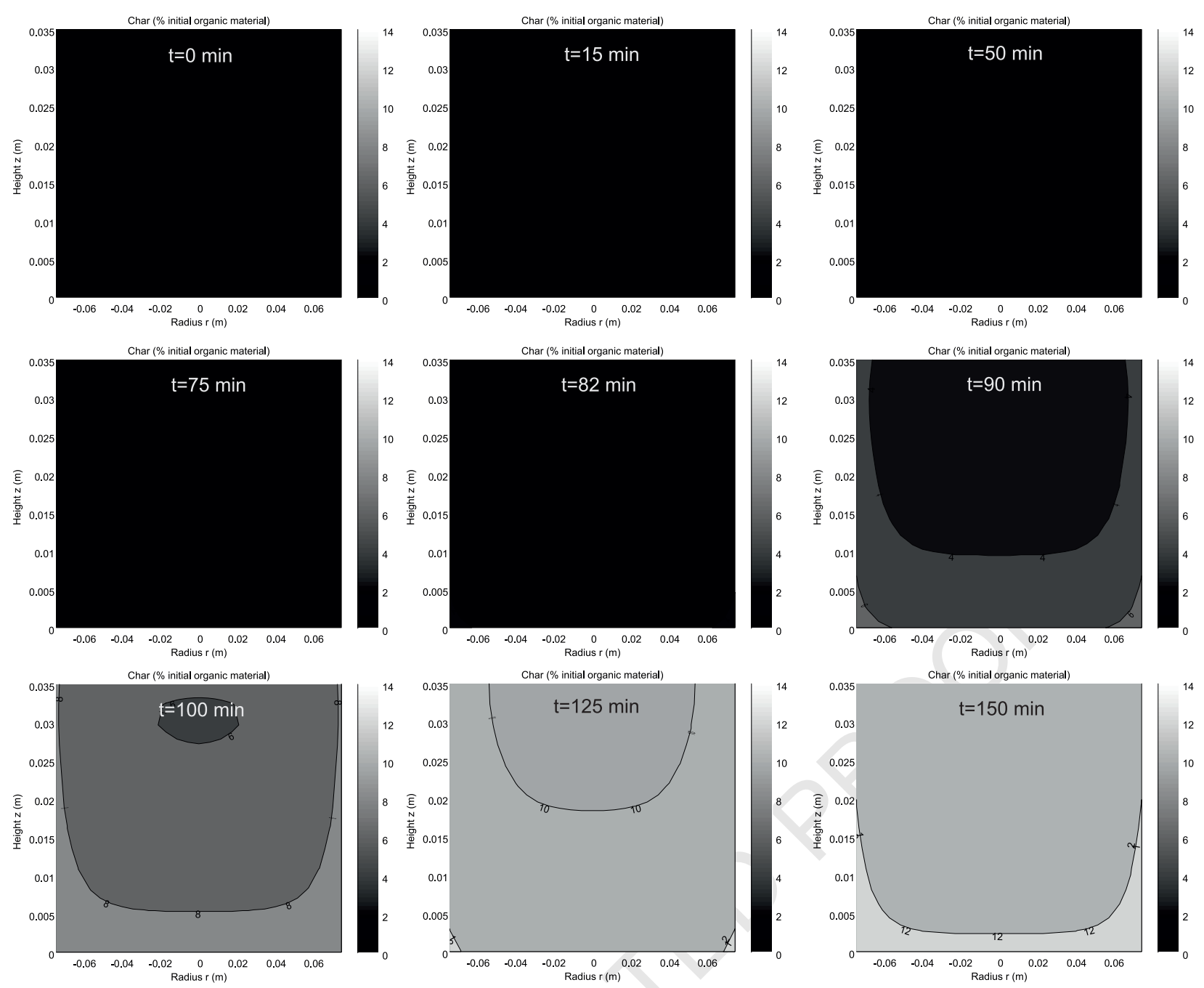

Figure 10. Evolution of the char content (in terms of percentage of the initial organic content) of the material within the basket as a function of space and time.

once this step is performed, the model will be used in order to determine the kinetics data of the reaction pathway that will be chosen to describe the pyrolysis of a sample. Not only will it allow to determine the value of the kinetics constant but also to determine the composition of the gases released during the pyrolysis reaction within the sample. This determination will have to be done so that the overall process balances the atomic content of the initial sample (C, H, O, N). Such a determination will be performed using the model coupled with an optimization algorithm in order to adjust the kinetics parameters of the model (kinetic constant, composition of the gas and intermediate involved in the reaction pathway) by minimizing (in the least square sense) the error between the mass loss, the differential mass loss, the evolution of the permanent gas composition and the average composition of condensable gases. These steps will be performed in some future work in order to build a database for determination of kinetics of pyrolysis of different samples.

\section{NOMENCLATURE}

$\alpha_{w} \quad$ activity of bound or liquid water (-)

$c_{p, \varphi} \quad$ Heat capacity of phase $\varphi(\mathrm{J} / \mathrm{kg} / \mathrm{K})$ $k_{\text {eff }} \quad$ Effective thermal conductivity $(\mathrm{W} / \mathrm{m} / \mathrm{K})$

$K_{\varphi} \quad$ Intrinsic permeability of phase $\varphi\left(\mathrm{m}^{2}\right)$

$K_{\varphi}^{r} \quad$ Relative permeability of phase $\varphi(-)$

$M \quad$ Moisture content (-)

$M_{f s p} \quad$ Moisture at fiber saturation point (-)

$M_{g} \quad$ Molar weight of the gas $(\mathrm{kg} / \mathrm{kmol})$

$\left\langle P_{g}\right\rangle^{\mathrm{g}} \quad$ Intrinsic liquid average pressure (Pa)

$\left\langle\rho_{l}\right\rangle^{l} \quad$ Intrinsic gas average pressure (Pa)

$P_{\text {sat }} \quad$ Vapour pressure of water $(\mathrm{Pa})$

$\langle T\rangle \quad$ Average temperature $(\mathrm{K})$

$\left\langle v_{l}\right\rangle_{j} \quad$ Average liquid velocity in direction $j(\mathrm{~m} / \mathrm{s})$

$\left\langle\dot{w}_{x}\right\rangle \quad$ Average rate of reaction of material $\mathrm{x}(\mathrm{kg}$ of $\mathrm{x} / \mathrm{m} 3$ of sample/s)

$\varepsilon_{\varphi} \quad$ Volume fraction of the phase $\varphi$ (m3 of phase $\varphi / \mathrm{m} 3$ of sample))

$\eta \quad$ Interpolation factor $(-)$

$\left\langle\rho_{l}\right\rangle^{g} \quad$ Intrinsic gas average mass concentration of species i $(\mathrm{kg}$ of $\mathrm{i} / \mathrm{m} 3$ of gas)

$\left\langle\rho_{\varphi}\right\rangle \quad$ Average mass concentration of phase $\varphi$ ( $\mathrm{kg}$ of phase $\varphi / \mathrm{m} 3$ of sample)

$\left\langle\rho_{\varphi}\right\rangle^{\varphi} \quad$ Intrinsic average mass concentration of phase $\varphi(\mathrm{kg}$ of phase $\varphi / \mathrm{m} 3$ of phase $\varphi$ ) 


\section{ACKNOWLEDGMENTS}

We Acknowledge the PCSTD (Pole de Calcul Scientifique et de Traitement des Donnees) of the Universite de Pau et des Pays de l'Adour (UPPA) for the computing facilities they provided us.

\section{REFERENCES}

[1] R. C. Brown, “Thermochemical Processing of Biomass”. 2011, John Wiley \& Sons.

[2] E. Hogan, J. Robert, G. Grassi, A. V. Bridgwater, "Biomass Thermal Processing". 1992, CPL Press.

[3] C. Di Blasi, Progress in Energy and Combustion Science 2008, 34, 47-90.

[4] C. Di Blasi, Progress in Energy and Combustion Science 1993, 19, 71-104.

[5] C. Higman, M. Van der Bugt, Gasification, 2nd edition. 2008, Elsevier.

[6] A. V. Bridgwater, "Fast Pyrolysis of biomass, A handbook, Volume 2". 2002, CPL Press.

[7] M. V de Velden, J. Baeyens, A. Brems, B. Janssens, R. Dewil, Renewable Energy 2010, 35, 232-242.

[8] G. Pantoleontos, P. Basinas, G. Skodras, P. Gramelis, J. D. Pinter, S. Topis, G. P. Sakellaropoulos, Fuel Processing Technology 2009, 90, 762-769.

[9] E. Grieco, G. Baldi, Chemical Engineering Science 2011, 66, 650-660.

[10] J. E. White, W. J. Catallo, B. L. Legendre, Journal of Analytical and Applied Pyrolysis 2011, 91, 1-33.

[11] C. Brage, Q. Yu, K. Sjostrom, Fuel 1996, 75, 213-219.

[12] D. Neves, H. Thunman, A. Matos, L. Tarelho, A. GomezBarea, Progress in Energy and Combustion Science 2011, 37, 611-630.

[13] K. Hashimoto, I. Hasegawa, J. Hayashil, K. Mae, Fuel 2011, 90, 104-112.

[14] T. Favarelli, A. Frassoldati, G. Migliavacca, E. Ranzi, Biomass and Bioenergy 2010, 34, 290-301.

[15] A. Dufour, B. Ouartassi, R. Bounaceur, A. Zoulalian, Chemical Engineering Research and Design 2011, 89, 1-11.

[16] E. Ranzi, A. Cuoci, T. Favarelli, A. Frassoldati, G. Migliavacca, S. Pierucci, S. Sommariva, Energy and Fuels 2008, 22, 4292-4300.

[17] D. K. Seo, S. S. Park, J. Hwang, T. Yu, Journal of Analytical and Applied Pyrolysis 2010, 89, 66-73.

[18] B. Peters, Fuel 2011, 90, 2301-2314.

[19] W. H. Chen, P. C. Kuo, Energy 2011, 36, 803-811.

[20] G. Duman, C. Okutucu, S. Ucar, R. Stahl, J. Yanik, Bioresource Technology 2011, 102, 1869-1878.

[21] S. Whitaker, Advances in Heat Transfer 1977, 13, 119-203.

[22] F. Golfier, B. D. Wood, L. Orgogozo, M. Quintard, M. Buès, Advances in Water Resources 2009, 32, 463-485.

[23] B. G. Lakatos, Chemical Engineering Science 2001, 56, 659666.

[24] A. A. M. Oliveira, M. Kaviany, Progress in Energy and Combustion Science 2001, 27, 523-545.

[25] M. Grønli, A theoretical and experimental study of the thermal degradation of biomass, Ph.D Thesis, The Norwegian University of Science and Technology, 1996.
[26] J. Ratte, F. Marias, J. Vaxelaire, P. Bernada, Journal of Hazardous Materials 2009, 170, 1023-1040.

[27] F. Shafizadeh, P. S. Chin, Chemical Aspects, ACS Symp. Ser. 1977, 43, 57-81.

[28] F. Thurner, U. Mann, Industrial Engineering Chemistry Process Design and Development 1981, 20, 482-488.

[29] J. J. M. Orfão, F. J. A. Antunes, J. L. Figueiredo, Fuel 1999, 78, 349-358.

[30] C. Di Blasi, M. Lanzetta, Journal of Analytical and Applied Pyrolysis 1997, 41, 287-303.

[31] C. A. Koufopanos, G. Mashio, The canadian journal of chemical engineering 1989, 67, 75-84.

[32] C. Casajus, Torrefaction de biomasses lingo cellulosiques, $\mathrm{PhD}$ Thesis. Université de Pau et des Pays de l'Adour, 2010.

[33] P. Bernada, F. Marias, A. Deydier, F. Couture, A. Fourcault, Waste and Biomass Valorisation 2012, In Press.

[34] P. Perre, I. Turner, The use of macroscopic equations to simulate heat mass transfer in porous media: some possibilities illustrated by a wide range of configurations that emphasise the role of internal pressure simultaneous heat, mass, momentum transfer in porous media: a theory of drying. in: Turner. Ian and Mujumdar. Arun, S Eds., Mathematical Modelling and Numerical Techniques in Drying Technology, Marcel Dekker, Inc, New York 1997.

[35] M. A. Stanish, G. S. Schajer, F. Kayihan, AIChE journal 1986, 32, 1301-1311.

[36] S. V. Patankar, Numerical Heat Transfer and Fluid Flow, Hemisphere Publishing Corporation, Washington, DC 1980.

[37] K. E. Brenan, S. L. Campbell, L. R. Petzold, Numerical solution of initial-value problems in Differential algebraic equations, Classics in Applied mathematics, SIAM, 1996.

[38] U. M. Ascher, L. R. Petzold, Computer methods for ordinary differential equations and Differential Algebraic equations, SIAM, 1998.

[39] A. Sargousse, J. M. Le Lann, X. Joulia, L. Jourda, Disco, un nouvel environnement de simulation orientée objet, In MOSIM'99, Modélisation et simulation des flux physiques et informationnels, 2ème Conférence Francophone de Modélisation et Simulation, Annecy, 1999.

Manuscript received February 12, 2014; revised manuscript received March 17, 2014; accepted for publication April 22, 2014. 
Required software to e-Annotate PDFs: Adobe Acrobat Professional or Adobe Reader (version 8.0 or above). (Note that this document uses screenshots from Adobe Reader $\mathrm{X}$ )

The latest version of Acrobat Reader can be downloaded for free at: http://get.adobe.com/reader/

Once you have Acrobat Reader open on your computer, click on the Comment tab at the right of the toolbar:

닙

This will open up a panel down the right side of the document. The majority of tools you will use for annotating your proof will be in the Annotations section, pictured opposite. We've picked out some of these tools below:

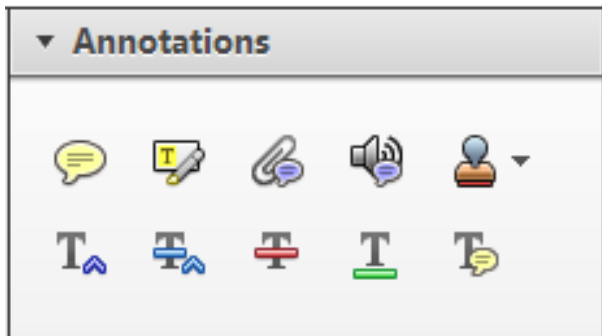

1. Replace (Ins) Tool - for replacing text.

Strikes a line through text and opens up a text box where replacement text can be entered.

\section{How to use it}

- Highlight a word or sentence.

- Click on the Replace (Ins) icon in the Annotations section.

- Type the replacement text into the blue box that appears.

Idard tramework for the analysis of $\mathrm{m}$ icy-Nevertheless, it also led to exog،

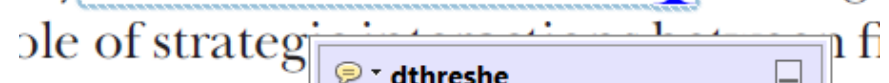
aber of comp 08/06/2011 15:58:17

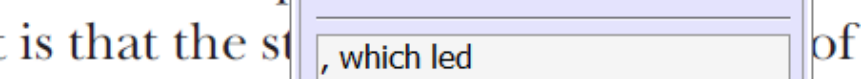
nain compo: be level, are exc nc

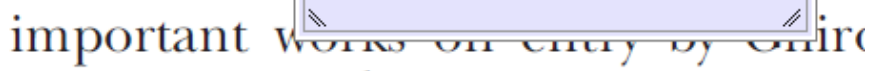
M heneferth) ${ }^{1}$ we anen the 'hlark $\mathrm{h}$

3. Add note to text Tool - for highlighting a section to be changed to bold or italic.

T Highlights text in yellow and opens up a text box where comments can be entered.

\section{How to use it}

- Highlight the relevant section of text.

- Click on the Add note to text icon in the Annotations section.

- Type instruction on what should be changed regarding the text into the yellow box that annears.

namic responses of mark ups ent with the VAR evidence

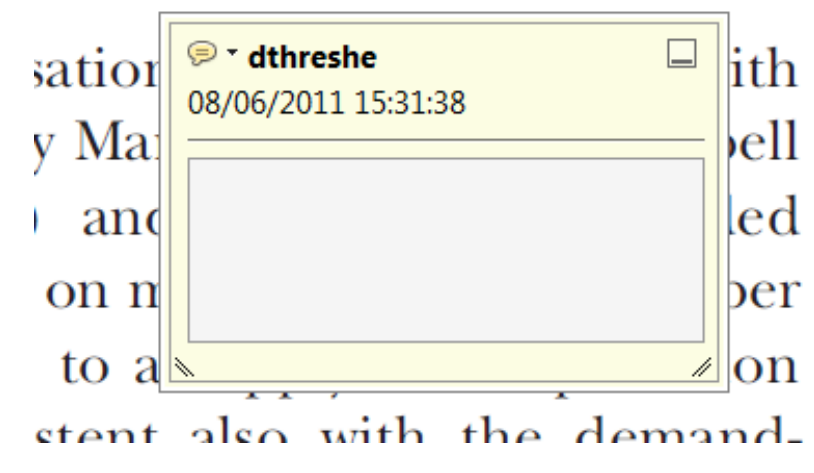

2. Strikethrough (Del) Tool - for deleting text.

Thikes a red line through text that is to be deleted.

\section{How to use it}

- Highlight a word or sentence.

- Click on the Strikethrough (Del) icon in the Annotations section.

there is no room tor extra prohts al c ups are zero and the number of ret) values are not determined by Blanchard and Kiyotaki (1987), rfect competition in general equilil ts of aggregate demand and supply lassical framework assuming monol eon an evorenois number of firms

\section{Add sticky note Tool - for making notes at} specific points in the text.

Marks a point in the proof where a comment needs to be highlighted.

\section{How to use it}

- Click on the Add sticky note icon in the Annotations section.

- Click at the point in the proof where the comment should be inserted.

- Type the comment into the yellow box that appears.

lallu allu suppiy silucks. hivst vi

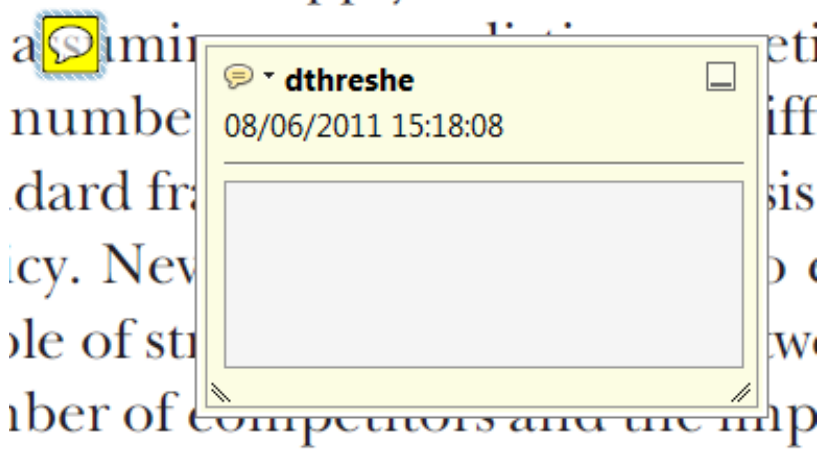

is that the structure of the secto 
5. Attach File Tool - for inserting large amounts of text or replacement figures.

Inserts an icon linking to the attached file in the appropriate pace in the text.

How to use it

- Click on the Attach File icon in the Annotations section.

- Click on the proof to where you'd like the attached file to be linked.

- Select the file to be attached from your computer or network.

- Select the colour and type of icon that will appear in the proof. Click OK.

E N D

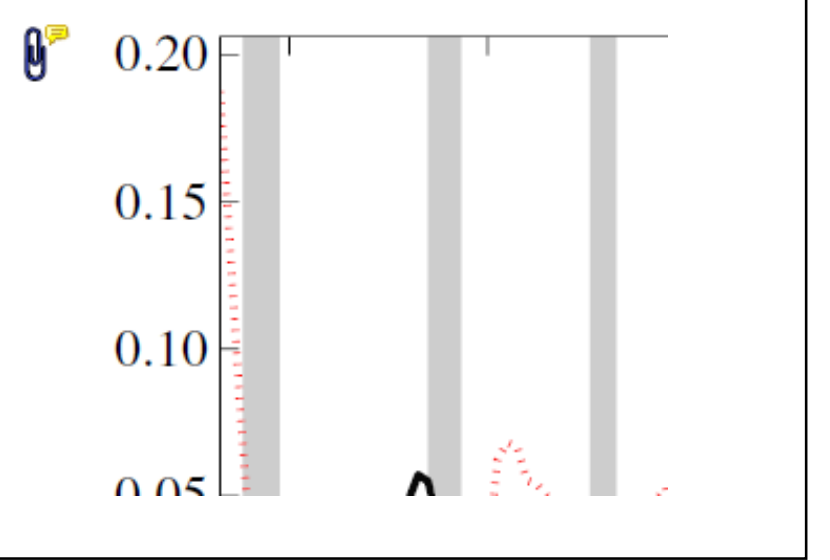

6. Add stamp Tool - for approving a proof if no corrections are required.

- Inserts a selected stamp onto an appropriate place in the proof

\section{How to use it}

- $\quad$ Click on the Add stamp icon in the Annotations section.

- $\quad$ Select the stamp you want to use. (The Approved stamp is usually available directly in the menu that appears).

- Click on the proof where you'd like the stamp to appear. (Where a proof is to be approved as it is, this would normally be on the first page).

of the Dusiness cycie, starting with the on perfect competition, constant ret

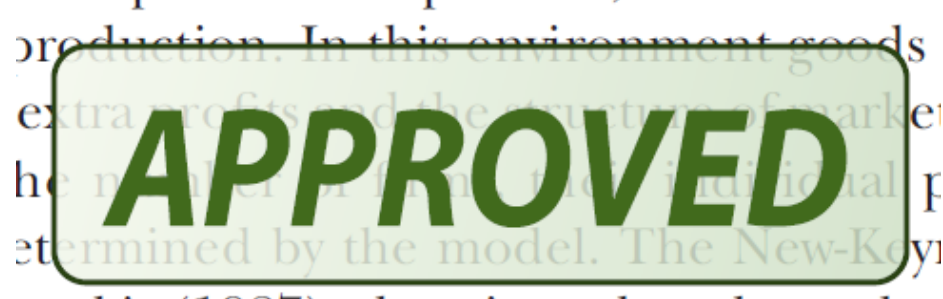

otaki (1987), has introduced produc general equilibrium models with nomin
- Drawing Markups

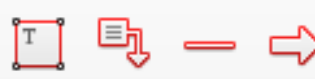

$0 \square \sqrt{6} D$

\section{How to use it}

- Click on one of the shapes in the Drawing Markups section.

- Click on the proof at the relevant point and draw the selected shape with the cursor.

- To add a comment to the drawn shape, move the cursor over the shape until an arrowhead appears.

- Double click on the shape and type any text in the red box that appears.
7. Drawing Markups Tools - for drawing shapes, lines and freeform annotations on proofs and commenting on these marks.

Allows shapes, lines and freeform annotations to be drawn on proofs and for comment to be made on these marks.

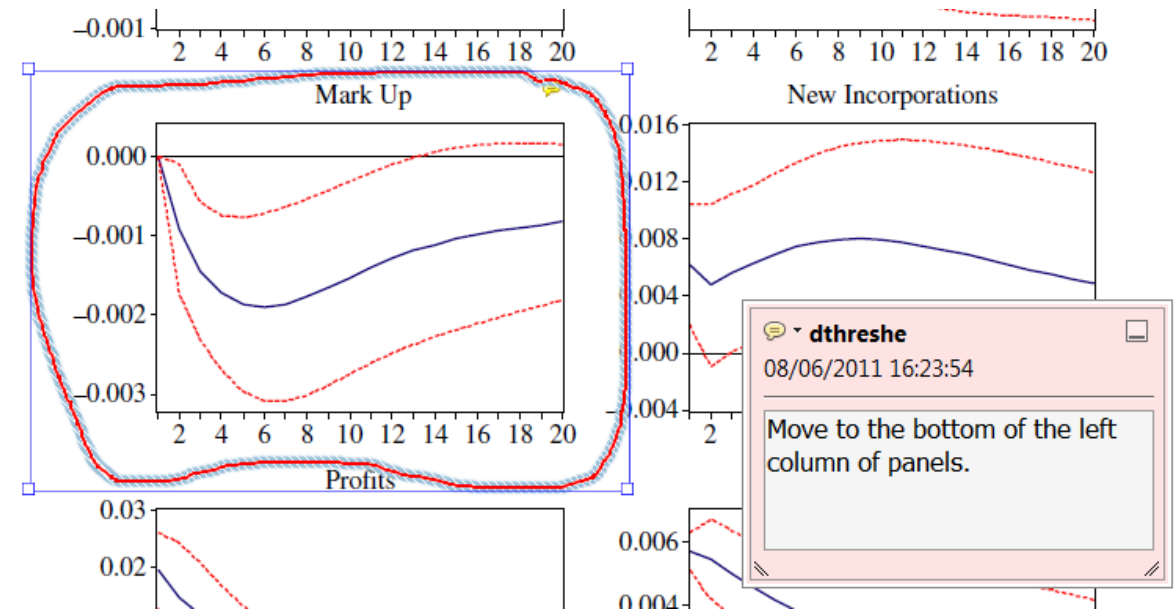

For further information on how to annotate proofs, click on the Help menu to reveal a list of further options:

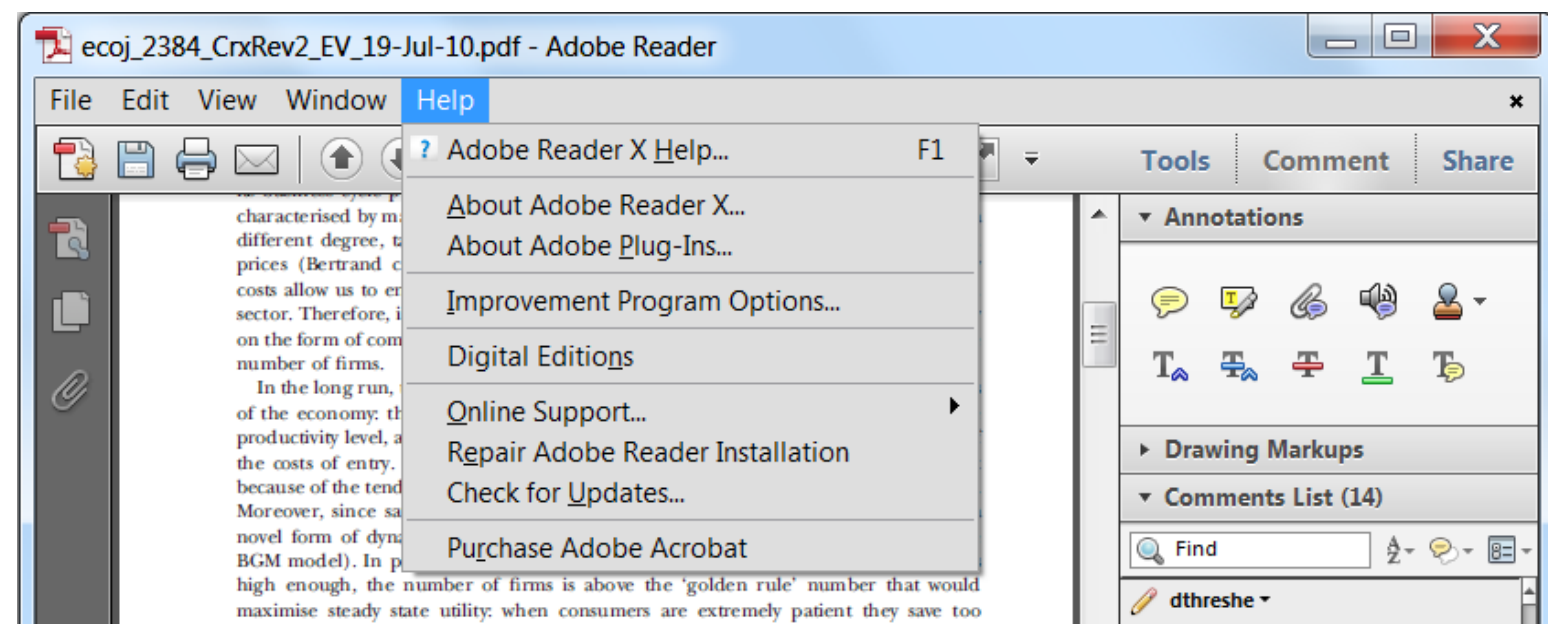




\section{(3)WILEY-BLACKWELL}

Additional reprint and journal issue purchases

Should you wish to purchase additional copies of your article, please click on the link and follow the instructions provided: https://caesar.sheridan.com/reprints/redir.php?pub=10089\&acro=CJCE?

Corresponding authors are invited to inform their co-authors of the reprint options available.

Please note that regardless of the form in which they are acquired, reprints should not be resold, nor further disseminated in electronic form, nor deployed in part or in whole in any marketing, promotional or educational contexts without authorization from Wiley. Permissions requests should be directed to mailto: permissionsus@wiley.com

For information about 'Pay-Per-View and Article Select' click on the following link: http://wileyonlinelibrary.com/ppv 